\title{
Numerical simulation of two coalescing turbulent forced plumes in linearly stratified fluids ${ }^{\circledR}$
}

Cite as: Phys. Fluids 31, 037111 (2019); https://doi.org/10.1063/1.5087534

Submitted: 02 January 2019 . Accepted: 08 March 2019 . Published Online: 28 March 2019

Yingzhong Lou (楼映中), Zhiguo He (贺治国) (D), Houshuo Jiang (蒋后硕), and Xiqiu Han (韩喜球)

\section{COLLECTIONS}

EP This paper was selected as an Editor's Pick
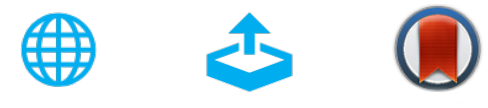

\section{ARTICLES YOU MAY BE INTERESTED IN}

Numerical study of the shear-thinning effect on the interaction between a normal shock wave and a cylindrical liquid column

Physics of Fluids 31, 043101 (2019); https://doi.org/10.1063/1.5083633

Harmonic linearized Navier-Stokes equation on describing the effect of surface roughness on hypersonic boundary-layer transition

Physics of Fluids 31, 034108 (2019); https://doi.org/10.1063/1.5086912

Formation of water in oil in water particles by drop impact on an oil layer

Physics of Fluids 31, 037107 (2019); https://doi.org/10.1063/1.5089001

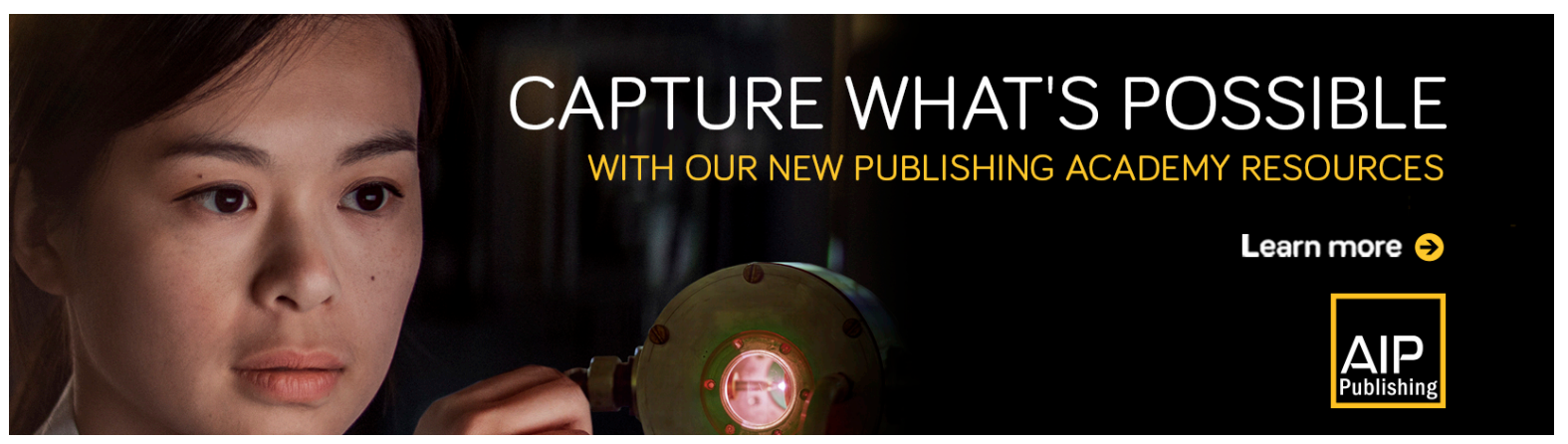




\title{
Numerical simulation of two coalescing turbulent forced plumes in linearly stratified fluids (루
}

\author{
Cite as: Phys. Fluids 31, 037111 (2019); doi: 10.1063/1.5087534 \\ Submitted: 2 January 2019 - Accepted: 8 March 2019 • \\ Published Online: 28 March 2019
}

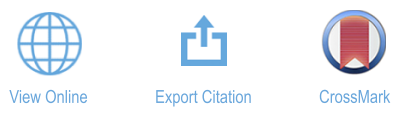

Yingzhong Lou (楼映中), ${ }^{3}$ Zhiguo He (贺治国), ${ }^{1,2, a)}$ (D) Houshuo Jiang (蒋后硕), ${ }^{3}$ and Xiqiu Han (韩喜球) ${ }^{1,4}$

\begin{abstract}
AFFILIATIONS
${ }^{1}$ Institute of Port, Coastal, and Offshore Engineering, Ocean College, Zhejiang University, Zhoushan 316021, China

${ }^{2}$ State Key Laboratory of Satellite Ocean Environment Dynamics, The Second Institute of Oceanography,

Ministry of Natural Resources, Hangzhou 310012, China

${ }^{3}$ Department of Applied Ocean Physics and Engineering, Woods Hole Oceanographic Institution, Woods Hole, Massachusetts 02543, USA

${ }^{4}$ Key Laboratory of Submarine Geoscience, State Oeanic Administration and Second Institute of Oceanography, Ministry of Natural Resources, Hangzhou 310012, China
\end{abstract}

a) Author to whom correspondence should be addressed: hezhiguo@zju.edu.cn

\begin{abstract}
A computational fluid dynamic model that can solve the Reynolds-averaged Navier-Stokes equations and the species transport equation is developed to simulate two coalescing turbulent forced plumes, which are released with initial momentum and buoyancy flux into a linearly stable stratified environment. The velocity fields, turbulence structures, and entrainment of two plumes with different source separations and source buoyancy fluxes are analyzed quantitatively, in comparison with a series of physical experiments. An empirical parameterization is proposed to predict the amplification of the maximum rise height of two coalescing forced plumes caused by superposition and mutual entrainment. The maximum values of both turbulent kinetic energy and turbulence dissipation rate decrease monotonically with the increase in source separation of the two turbulent plumes. However, the trajectory of the maximum turbulent viscosity attained in the plume cap region presents two notable enhancements. This variation may be attributed to the turbulence transported from the touching region and the strong mixing around the neutrally buoyant layer between two plumes, while the mixing is caused by the lateral convection and the rebound after overshooting. The plume entrainment coefficient in near vent stems has a positive relationship with the source Richardson number. A transition of flow regimes to plume-like flows would occur when the contribution of initial momentum is important. The entrainment coefficient will decrease in the touching region of two plumes due to mutual entrainment, while the superposition of plumes can lead to distortion of the boundary of plume sectors.
\end{abstract}

Published under license by AIP Publishing. https://doi.org/10.1063/1.5087534

\section{INTRODUCTION}

Forced plumes at different temporal and spatial scales are quite widespread in nature and industrial fields such as volcanic eruptions, ${ }^{1}$ upwelling flows of meltwater at the base of glaciers, ${ }^{2,3}$ and discharges of treated wastewaters into coastal areas. ${ }^{4,5}$ This flow regime, which is closely associated with both inertia and buoyancy, is of great interest. For example, the studies of submarine hydrothermal plumes have great significance to a series of cross subjects, ${ }^{6,7}$ including polymetallic metallogenesis, ${ }^{8-10}$ chemoautotrophic microbial communities, ${ }^{17-13}$ and ocean-scale biogeochemical budgets of elements. ${ }^{14-16}$
Based on the "entrainment assumption," Morton et al. ${ }^{17}$ derived an integral model to theoretically describe an axisymmetric Boussinesq plume, named the Morton-Taylor-Turner (MTT) model. The model suggested that the maximum rise height of a plume in a linearly stratified fluid could be expressed as a function of the source buoyancy flux and the background buoyancy frequency, and it was extended to the regime of forced plumes by Morton. ${ }^{18}$ Moreover, many numerical simulations, physical experiments, and analytical solutions have been conducted to investigate the dynamics of buoyant plumes, ${ }^{19-31}$ and the research for the plumes generated by thermal convection is also abundant. ${ }^{32-36}$ In particular, Lavelle ${ }^{37}$ described the behavior of hydrothermal plumes in a rotating 
stratified environment by a convection model, and he showed the model to be feasible when simulating the characteristics in the plume cap region and the circulation in the surrounding environment. Pham et al. ${ }^{38}$ investigated turbulent thermal plumes by direct numerical simulation (DNS) and large eddy simulation (LES). They pointed out that areas near the source should be simulated carefully to yield reasonable flow structures. Tao et al. ${ }^{39}$ simulated hydrothermal plumes in a static and dynamic environment using a 3D time-dependent approach, and they suggested that transported turbulence could cause the damping of the oscillation around the neutrally buoyant layer. Jiang and Breier ${ }^{40}$ investigated the effect of vent properties and near vent physical conditions on submarine hydrothermal plumes and derived several characteristic scalings using a simulation. Their results implied that the classical entrainment assumption is not applicable above the neutrally buoyant layer. Zhang et al. ${ }^{41}$ established a tracer transport model considering density variation to simulate the propagation of a buoyant jet interacting with a boundary in a stratified fluid. The model was used to investigate the velocity and density structures of jets regarding the Reynolds number and the ratios of the characteristic momentum length to the buoyancy length as variables. Pant and Bhattacharya ${ }^{42}$ made an attempt to estimate the entrainment coefficient for forced jets according to the energy-consistent approach, and they conducted a series of large eddy simulations to validate the generated model.

Furthermore, the coalescence of multiple plumes widely exists in natural and artificial systems, e.g., multiple hydrothermal plumes released from clustered submarine vents, ${ }^{43-46}$ moist air discharged from adjacent cooling towers and condensed by entraining cold air, ${ }^{47,48}$ and air circulations caused by multiple heat sources located at various positions within a naturally ventilated building. ${ }^{49,50}$ However, there are relatively few studies focusing on interactions between multiple plumes, which include semi-empirical models, ${ }^{51-54}$ numerical simulations, ${ }^{55}$ and experimental observations. $^{56-62}$ In particular, Bornoff and Mokhtarzadeh-Dehghan ${ }^{47}$ developed a finite-volume model to simulate two cooling tower plumes in a neutral cross-flow using a low Reynolds number $k-\varepsilon$ turbulence model. Mokhtarzadeh-Dehghan et al. ${ }^{63}$ simulated plumes from two cooling towers and conducted a parameter sensitivity analysis around different turbulence models. Their model successfully reproduced a pair of counter-rotating vortices and obtained a prediction of plume rise height that agreed with the experimental and theoretical data. Kaye and Linden ${ }^{64}$ proposed a model that focused on merging plume pairs in a uniform environment. By describing plume interactions as the deflection of plume axes, the model showed a good agreement with observations. Cenedese and Linden ${ }^{65}$ extended that model into a region in which two plumes are touching but not merged, and they proposed an "effective" entrainment coefficient to parameterize the total volume flux of the two coalescing plumes. Lai and $\mathrm{Lee}^{4}$ used a semi-analytical model to simulate multiple buoyant jets in a uniform ambience, which also follows the assumption that the flow field is irrotational and can be modeled by a distribution of point sinks along each trajectory while the velocity distribution is Gaussian and additive. Rooney ${ }^{66,67}$ developed a series of models to describe multiple buoyant plumes arranged along a straight line or around a circle in an unstratified environment. By considering the distortion of plume cross sections and closing the governing equations with an adjusted entrainment assumption, the models could provide appropriate similarity scalings in the near and far fields and could present an evolution of the plume volume flux that compares favorably with experimental results for two merging plumes. Most recently, Kaye and Cooper ${ }^{68}$ focused on the turbulent plumes generated by vertically distributed sources in a uniform surrounding, and they discussed the effects of wall shear stress and source

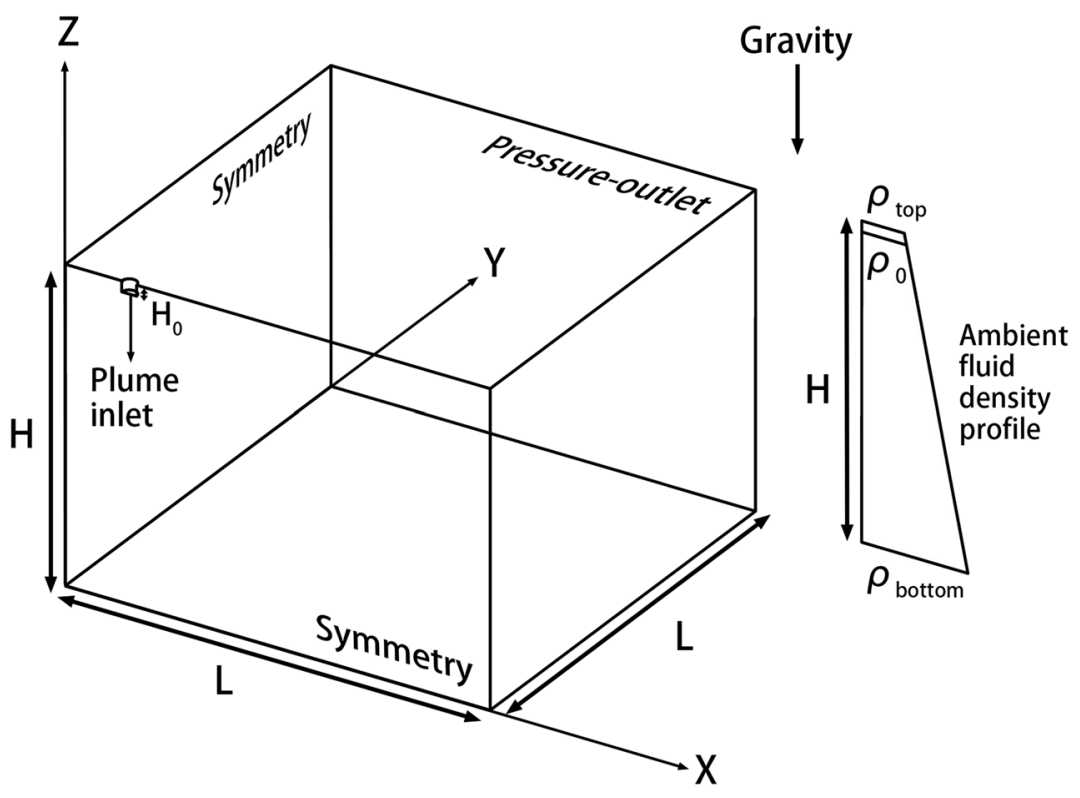

FIG. 1. Schematic of the computational domain and the boundary conditions except for walls. 

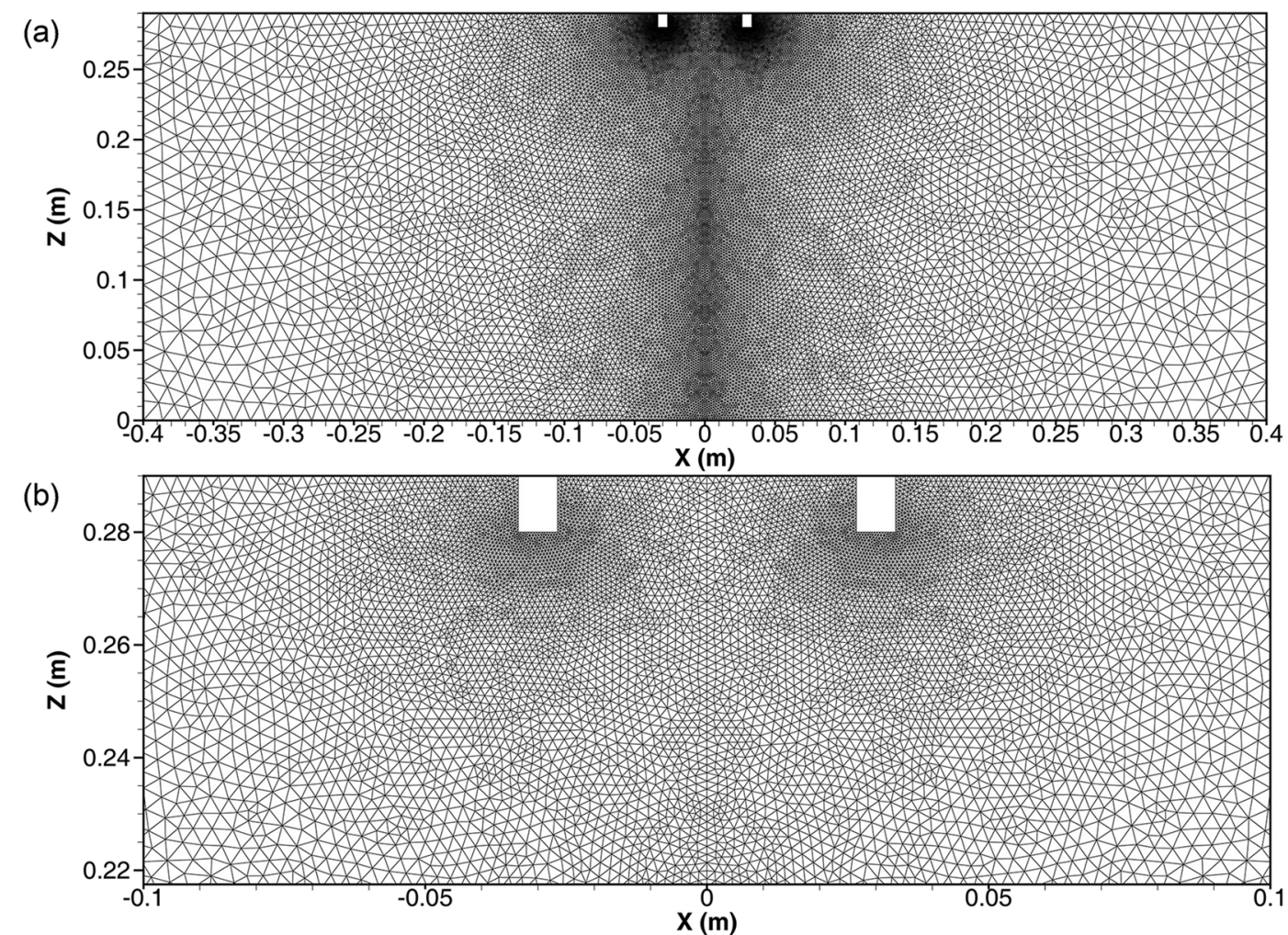

FIG. 2. Grids in the XZ plane of the CFD model: (a) the whole computational domain and (b) the near vent region.

TABLE I. Summary of the parameters for the numerical experiments (SI units).

\begin{tabular}{|c|c|c|c|c|c|c|c|c|c|}
\hline Cases & $L$ & $Q_{\text {exit }}$ & $B_{\text {exit }}$ & $N$ & $\rho_{0}$ & $\rho_{\text {exit }}$ & $R i$ & $R e_{p}$ & $R e_{c}$ \\
\hline 1 & $1.5 \times 10^{-2}$ & $3.71 \times 10^{-6}$ & $9.83 \times 10^{-7}$ & 0.90 & 998.82 & 1025.8 & 0.421 & $6.91 \times 10^{2}$ & $2.86 \times 10^{4}$ \\
\hline 2 & $3.0 \times 10^{-2}$ & $3.71 \times 10^{-6}$ & $9.95 \times 10^{-7}$ & 0.87 & 998.84 & 1026.1 & 0.423 & $6.91 \times 10^{2}$ & $2.86 \times 10^{4}$ \\
\hline 3 & $6.0 \times 10^{-2}$ & $3.70 \times 10^{-6}$ & $1.00 \times 10^{-6}$ & 0.86 & 999.33 & 1026.1 & 0.420 & $6.89 \times 10^{2}$ & $2.85 \times 10^{4}$ \\
\hline 4 & $9.0 \times 10^{-2}$ & $3.71 \times 10^{-6}$ & $1.00 \times 10^{-6}$ & 0.86 & 999.51 & 1026.1 & 0.417 & $6.91 \times 10^{2}$ & $2.86 \times 10^{4}$ \\
\hline 5 & $1.2 \times 10^{-1}$ & $3.70 \times 10^{-6}$ & $9.73 \times 10^{-7}$ & 0.88 & 998.89 & 1025.7 & 0.420 & $6.89 \times 10^{2}$ & $2.85 \times 10^{4}$ \\
\hline 6 & $1.5 \times 10^{-2}$ & $5.69 \times 10^{-6}$ & $1.53 \times 10^{-6}$ & 0.89 & 1000.8 & 1028.3 & 0.276 & $1.06 \times 10^{3}$ & $4.40 \times 10^{4}$ \\
\hline 7 & $3.0 \times 10^{-2}$ & $5.71 \times 10^{-6}$ & $1.53 \times 10^{-6}$ & 0.87 & 998.77 & 1026.1 & 0.275 & $1.06 \times 10^{3}$ & $4.40 \times 10^{4}$ \\
\hline 8 & $6.0 \times 10^{-2}$ & $5.70 \times 10^{-6}$ & $1.53 \times 10^{-6}$ & 0.88 & 998.79 & 1026.1 & 0.276 & $1.06 \times 10^{3}$ & $4.40 \times 10^{4}$ \\
\hline 9 & $9.0 \times 10^{-2}$ & $5.70 \times 10^{-6}$ & $1.54 \times 10^{-6}$ & 0.86 & 998.75 & 1026.3 & 0.276 & $1.06 \times 10^{3}$ & $4.40 \times 10^{4}$ \\
\hline 10 & $1.2 \times 10^{-1}$ & $5.70 \times 10^{-6}$ & $1.53 \times 10^{-6}$ & 0.87 & 998.77 & 1026.1 & 0.276 & $1.06 \times 10^{3}$ & $4.40 \times 10^{4}$ \\
\hline 11 & $1.5 \times 10^{-2}$ & $8.99 \times 10^{-6}$ & $2.40 \times 10^{-6}$ & 0.91 & 998.84 & 1026.1 & 0.174 & $1.67 \times 10^{3}$ & $6.93 \times 10^{4}$ \\
\hline 12 & $3.0 \times 10^{-2}$ & $9.02 \times 10^{-6}$ & $2.40 \times 10^{-6}$ & 0.90 & 998.82 & 1025.9 & 0.173 & $1.68 \times 10^{3}$ & $6.96 \times 10^{4}$ \\
\hline 13 & $6.0 \times 10^{-2}$ & $9.02 \times 10^{-6}$ & $2.35 \times 10^{-6}$ & 0.89 & 998.81 & 1025.4 & 0.172 & $1.68 \times 10^{3}$ & $6.95 \times 10^{4}$ \\
\hline 14 & $9.0 \times 10^{-2}$ & $9.02 \times 10^{-6}$ & $2.41 \times 10^{-6}$ & 0.90 & 998.82 & 1026.1 & 0.174 & $1.68 \times 10^{3}$ & $6.96 \times 10^{4}$ \\
\hline 15 & $1.2 \times 10^{-1}$ & $9.01 \times 10^{-6}$ & $2.36 \times 10^{-6}$ & 0.88 & 998.79 & 1025.5 & 0.172 & $1.68 \times 10^{3}$ & $6.95 \times 10^{4}$ \\
\hline 16 & Infinity & $3.71 \times 10^{-6}$ & $9.83 \times 10^{-7}$ & 0.90 & 998.82 & 1025.8 & 0.421 & $6.91 \times 10^{2}$ & $2.86 \times 10^{4}$ \\
\hline 17 & Infinity & $5.69 \times 10^{-6}$ & $1.53 \times 10^{-6}$ & 0.89 & 1000.8 & 1028.3 & 0.276 & $1.06 \times 10^{3}$ & $4.40 \times 10^{4}$ \\
\hline 18 & Infinity & $8.99 \times 10^{-6}$ & $2.40 \times 10^{-6}$ & 0.91 & 998.84 & 1026.1 & 0.174 & $1.67 \times 10^{3}$ & $6.93 \times 10^{4}$ \\
\hline
\end{tabular}


conditions at different heights combining with previous experiments. Considering both open and confined cases, they analyzed the potential impact of these effects on plume entrainment and detrainment. Zhou et al. ${ }^{69}$ modeled a series of two plane jets with different source separations by DNS to investigate the spatial structure of both mean and fluctuating velocities when ambient streams exist, and the results suggest that the relationship between the merging distance and the source separation could be linear.
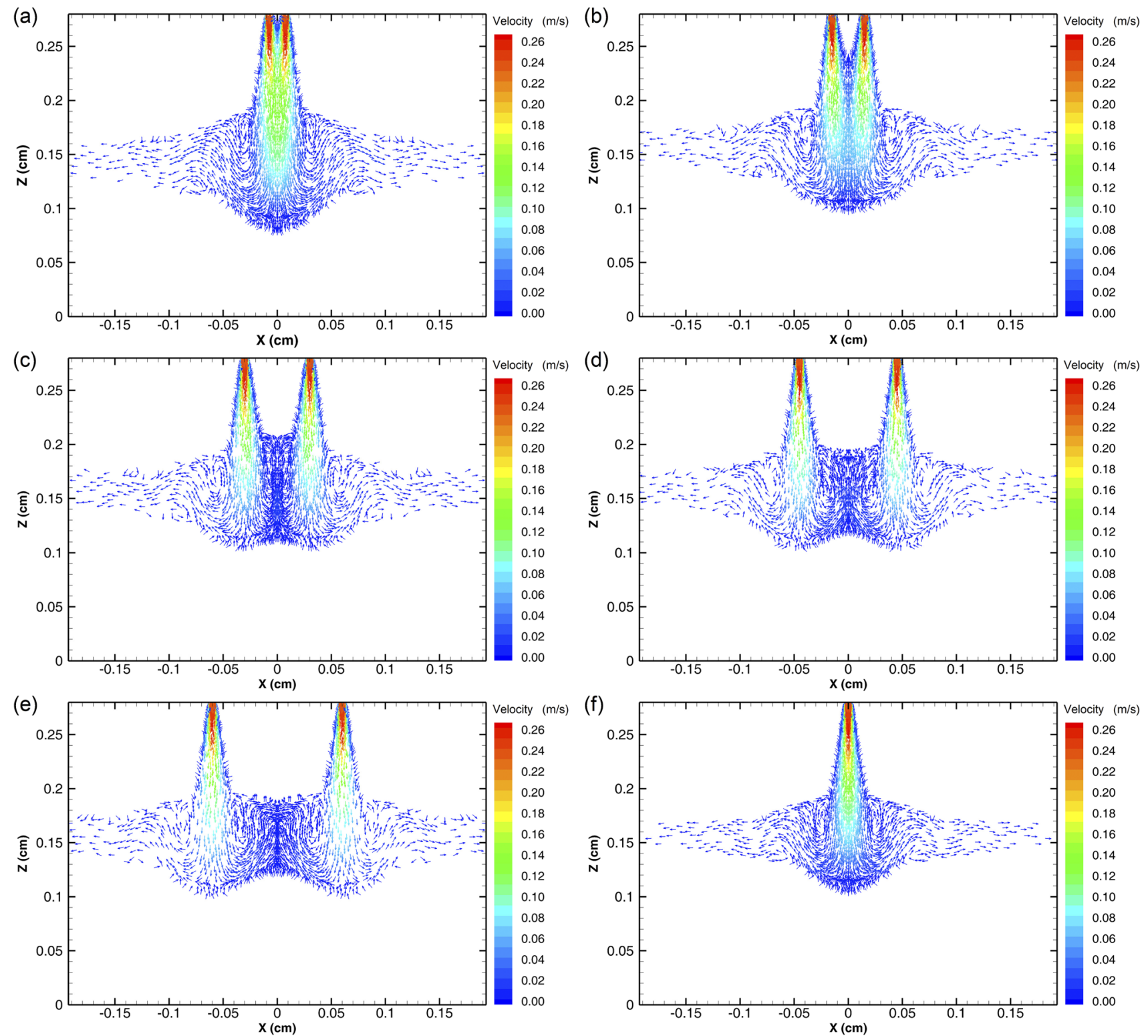

FIG. 3. Distribution of the velocity fields (colored vectors with uniform vector length) of the two plumes for cases $11-15$ [(a)-(e)] and the single plume counterpart for case $18(\mathrm{f})$ 
The numerical methods are described in Sec. II. The results of the model are described and compared with that observed in physical experiments in Sec. III. Finally, our conclusions are summarized in Sec. IV.

\section{METHODS}

Our numerical model was performed with ANSYS Fluent and was based on the finite-volume method. The main governing

(a)

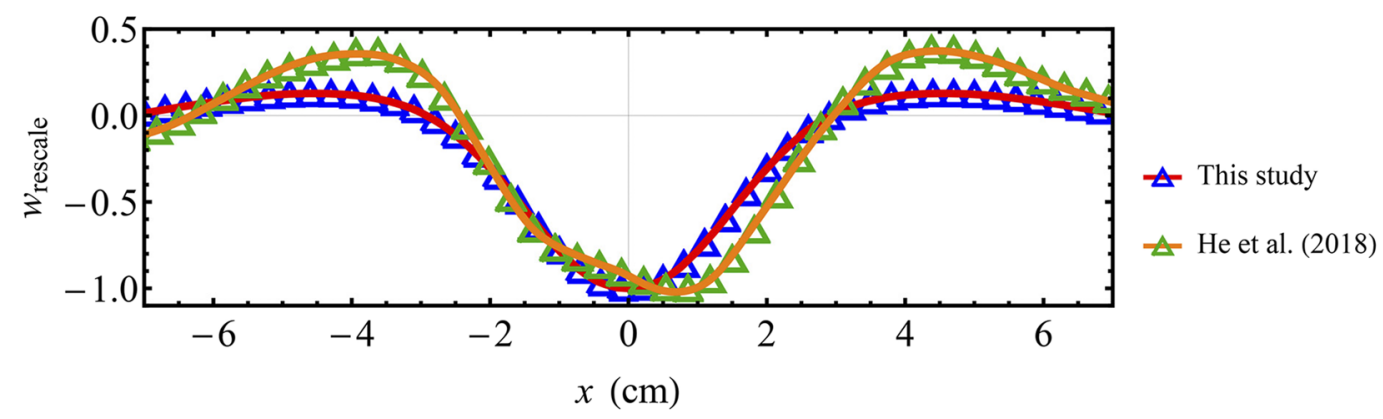

(b)

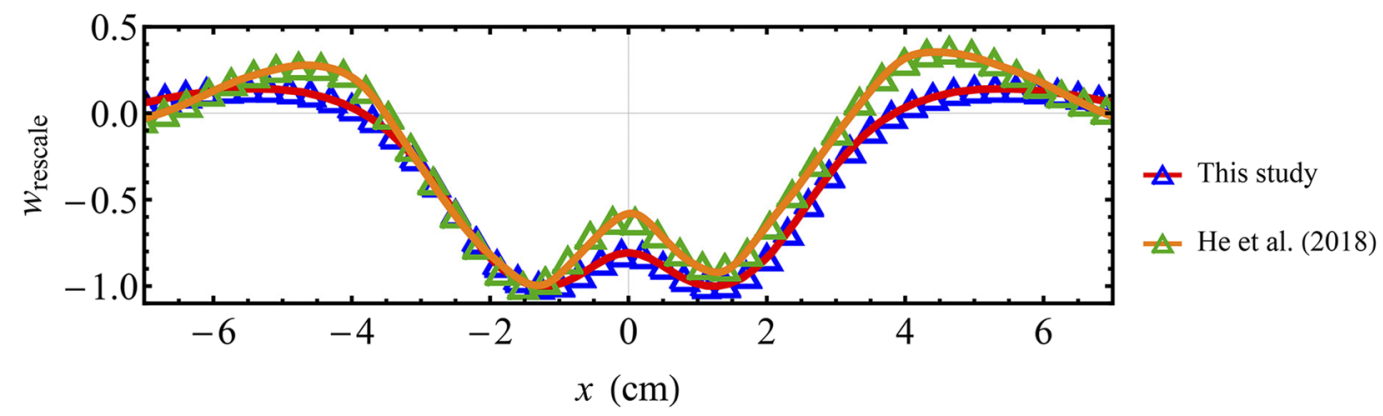

(c)

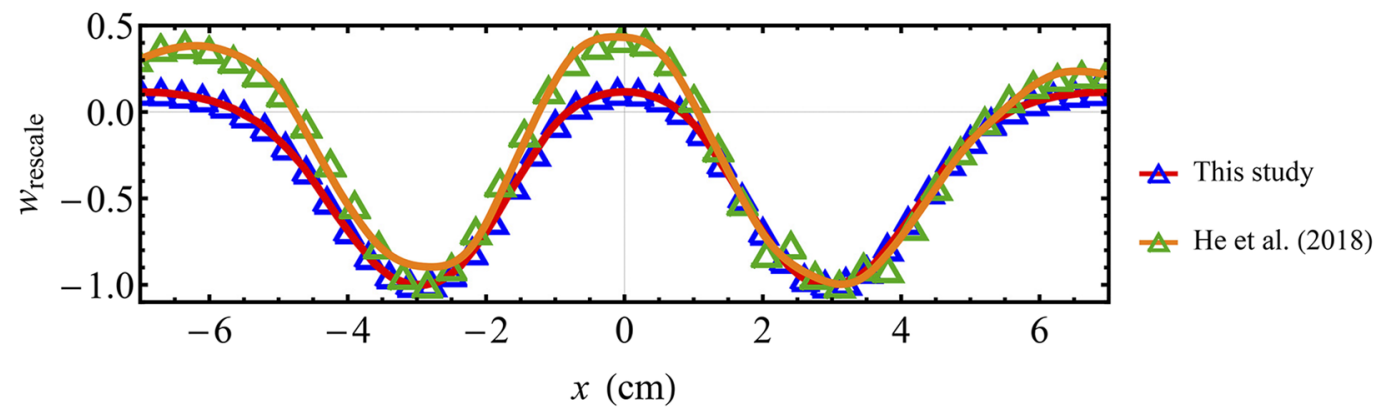

(d)

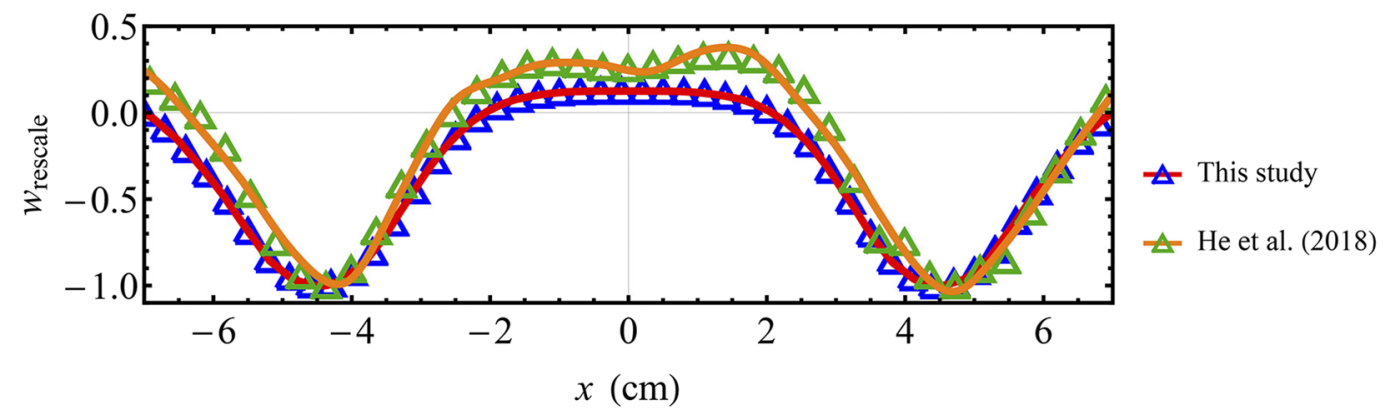

FIG. 4. Radial profiles of the normalized vertical velocity in the neutrally buoyant layer for cases 11-14 [(a)-(d)]. 
equations are provided in Appendix A, and the details of numerical scheme for the finite-volume solver are provided in Appendix B. Specifically, the species transport model is employed since there are two completely miscible liquids involved in the flow. Taking the salt water and fresh water as two component species, the species transport model in ANSYS Fluent can be used to simulate the mixing processes without reactions by solving the convection and diffusion equation.

The computational domain of the model is a cuboid $(40 \times$ $40 \times 29 \mathrm{~cm}$ ) with two symmetrical planes (i.e., XZ and YZ coordinate planes), i.e., a quarter of that used in the laboratory experiments. ${ }^{62}$ The schematic of the computational domain and the boundary conditions, except for walls, are shown in Fig. 1, where $H$ is the full depth of the computational domain (i.e., $29 \mathrm{~cm}$ ) and $H_{0}$ is the depth of the plume inlet below the surface and is taken as $1 \mathrm{~cm}$ here.

The domain is fully discretized by tetrahedron cells. Specifically, the vent diameter $(7 \mathrm{~mm})$ is evenly divided into 15 segments according to suggestions given in previous research, ${ }^{70,71}$ and the central axis (i.e., Z-axis) is divided into 200 segments to provide a sufficient accuracy to quantify the plume cap regions. Other cells are stretched at a constant rate of 1.03 from the vent orifice to the domain boundaries, as shown in Fig. 2.

According to the MTT model, a process of two forced plumes is uniquely determined by the buoyancy frequency $N$, the source buoyancy flux $B_{\text {exit }}$, the source Richardson number $R i$, the source volume flux $Q_{\text {exit }}$, and the source separation $L$. These can be expressed as

$$
\begin{gathered}
N=\sqrt{-\frac{g}{\rho_{\text {top }}} \frac{d \rho(Z)}{d Z},} \\
B_{\text {exit }}=g \frac{\rho_{\text {exit }}-\rho_{0}}{\rho_{0}} Q_{\text {exit }}, \\
R i=\frac{Q_{\text {exit }} B_{\text {exit }}{ }^{1 / 2}}{M_{\text {exit }}{ }^{5 / 4}},
\end{gathered}
$$

where $g$ is the gravitational acceleration; $\rho(Z), \rho_{t o p}$, and $\rho_{0}$ are the density of ambient fluids at an arbitrary height, at the top of the domain, and near vent region, respectively; $\rho_{\text {exit }}$ and $M_{\text {exit }}$ are the source density and momentum flux. Also, the initial setup of the density profile can be expressed as

$$
\rho(Z)=\rho_{0} \frac{1+N^{2}(H-Z) / g}{1+N^{2} H_{0} / g} .
$$

In accordance with the conditions of the laboratory experiments, ${ }^{62}$ the main parameters of the numerical experiments for all eighteen cases are selected and listed in Table I. In particular, the last three, i.e., cases 16-18, are all single-plume cases that serve as control groups.

The flow Reynolds numbers based the plumes scale $R e_{p}$ and the container scale $R e_{c}$ are shown in Table I. Specifically, the vent diameter (i.e., $7 \mathrm{~mm}$ ) and the initial plume velocity are chosen as the characteristic length and velocity for $R e_{p}$, respectively, while the

\begin{tabular}{|c|c|c|c|c|c|c|c|}
\hline \multirow[b]{2}{*}{ Cases } & \multirow[b]{2}{*}{$L$} & \multicolumn{3}{|c|}{ Numerical model } & \multicolumn{3}{|c|}{ Physical experiments } \\
\hline & & $\overline{Z_{\text {max }}^{\prime}}$ & $Z_{\max }$ & $\zeta$ & $\overline{Z_{\text {max }}^{\prime}}$ & $Z_{\max }$ & $\zeta$ \\
\hline 1 & 1.5 & 14.60 & 12.81 & 1.139 & 15.58 & 13.73 & 1.135 \\
\hline 2 & 3.0 & 12.68 & 12.74 & 0.995 & 15.08 & 13.65 & 1.105 \\
\hline 3 & 6.0 & 12.83 & 12.77 & 1.005 & 13.92 & 13.69 & 1.017 \\
\hline 4 & 9.0 & 12.89 & 12.86 & 1.002 & 13.90 & 13.79 & 1.008 \\
\hline 5 & 12 & 12.92 & 13.00 & 0.994 & 13.97 & 13.93 & 1.003 \\
\hline 6 & 1.5 & 16.72 & 14.43 & 1.159 & 17.16 & 15.46 & 1.110 \\
\hline 7 & 3.0 & 15.37 & 14.68 & 1.047 & 16.95 & 15.74 & 1.077 \\
\hline 8 & 6.0 & 14.86 & 14.55 & 1.021 & 15.95 & 15.58 & 1.024 \\
\hline 9 & 9.0 & 15.38 & 14.83 & 1.037 & 15.97 & 15.90 & 1.004 \\
\hline 10 & 12 & 14.98 & 14.68 & 1.021 & 15.75 & 15.73 & 1.001 \\
\hline 11 & 1.5 & 18.81 & 15.88 & 1.184 & 18.98 & 17.03 & 1.115 \\
\hline 12 & 3.0 & 17.37 & 16.02 & 1.084 & 18.58 & 17.16 & 1.083 \\
\hline 13 & 6.0 & 16.62 & 16.07 & 1.035 & 17.59 & 17.23 & 1.021 \\
\hline 14 & 9.0 & 16.69 & 16.03 & 1.041 & 17.41 & 17.18 & 1.013 \\
\hline 15 & 12 & 16.93 & 16.22 & 1.044 & 17.33 & 17.39 & 0.997 \\
\hline 16 & Infinity & 12.81 & 12.81 & 1.000 & $\ldots$ & $\ldots$ & 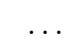 \\
\hline 17 & Infinity & 14.83 & 14.43 & 1.028 & $\ldots$ & . & \\
\hline 18 & Infinity & 16.52 & 15.88 & 1.040 & $\ldots$ & $\ldots$ & $\ldots$ \\
\hline
\end{tabular}
full depth of the container (i.e., $29 \mathrm{~cm}$ ) and a half of the initial
TABLE II. Summary of the maximum plume rise heights and the related results for all cases (unit of length: $\mathrm{cm}$ ).

velocity are chosen for $R e_{c}$. According to the stability study conducted by Tveitereid and Riley, ${ }^{72}$ both of these two flow Reynolds numbers and the turbulence spectra obtained by the corresponding

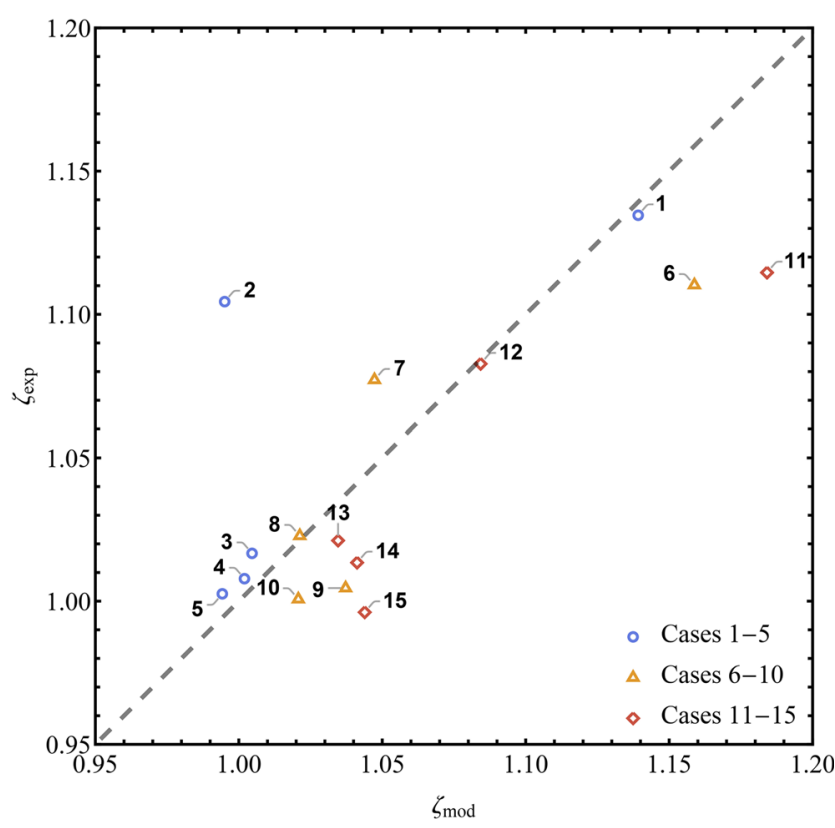

FIG. 5. A comparison of the amplification coefficient given by the numerical mode $\left(\zeta_{\bmod }\right)$ and the physical experiments $\left(\zeta_{\exp }\right)$. 
experiments $^{62}$ imply the existence of turbulence. Hence, the realizable $k-\varepsilon$ model (see Appendix A for detail) is introduced in the simulation. The robustness and accuracy of the $k-\varepsilon$ model for a wide range of turbulent flows have been validated by extensive simulations. Besides, as the main flow is sufficiently away from the wall, the main propagation of plume with a high enough Reynolds number can be regarded as fully turbulent flows. Moreover, the $k-\varepsilon$ model has been shown to be feasible in general for modeling forced plumes in a linearly stratified environment, such as submarine hydrothermal plumes ${ }^{40}$ and vertical buoyant wall jets. ${ }^{41}$ Therefore, the two plumes could be reasonably modeled by the $k-\varepsilon$ model in this study.
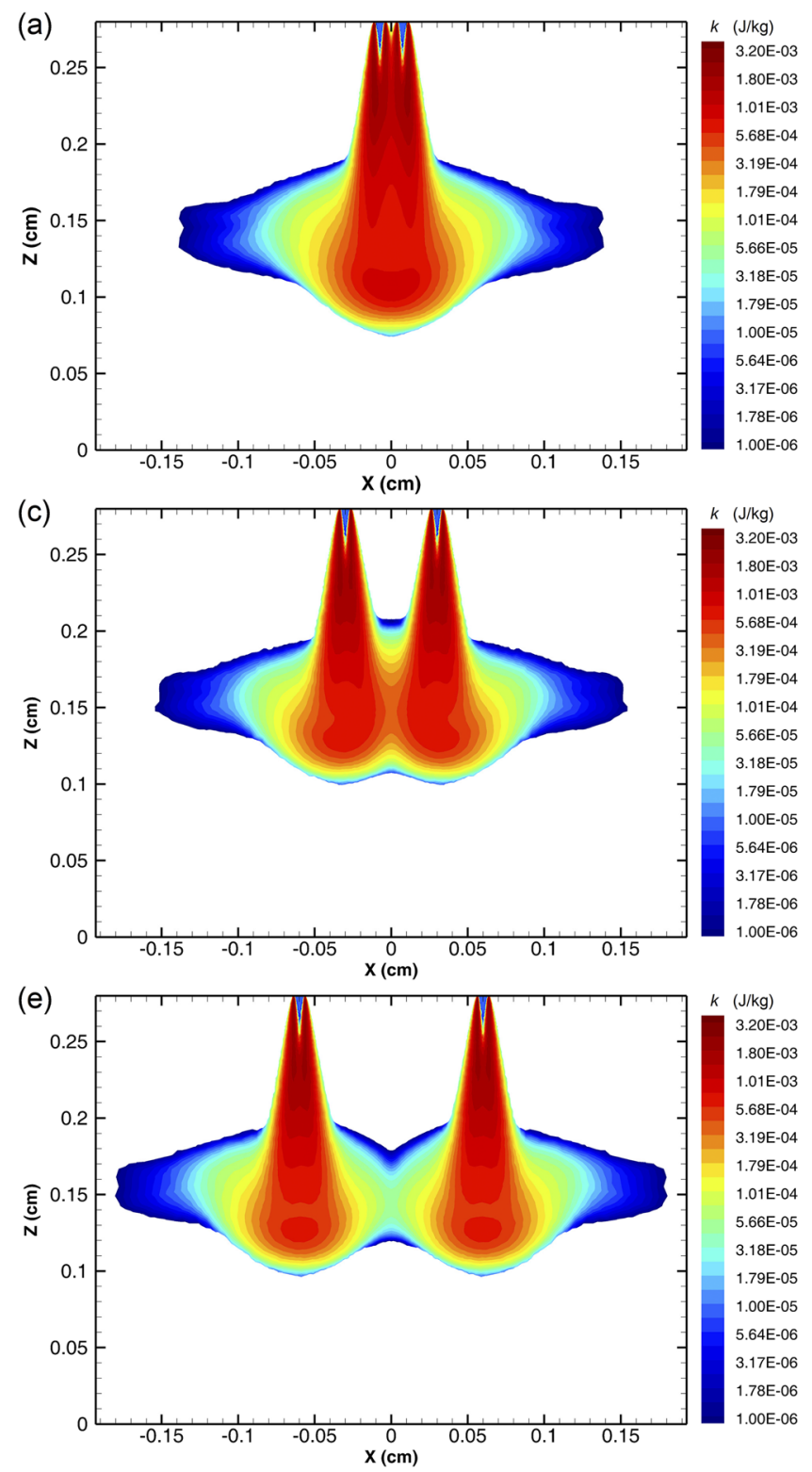

For each case, the total simulation time is $4 t^{*}$, where $t^{*}=2 \pi / N$ is the buoyancy time scale. In addition, a conservative time step of $0.002 \mathrm{~s}$ was selected to ensure that the numerical calculation could be converged within 20 iterations at each time step.

\section{RESULTS AND DISCUSSION}

\section{A. Plume velocity field}

Several typical velocity fields of the two coalescing forced plumes with a large source buoyancy flux, i.e., the results for cases 11-15, are shown in Fig. 3, where the result for case 18 is also
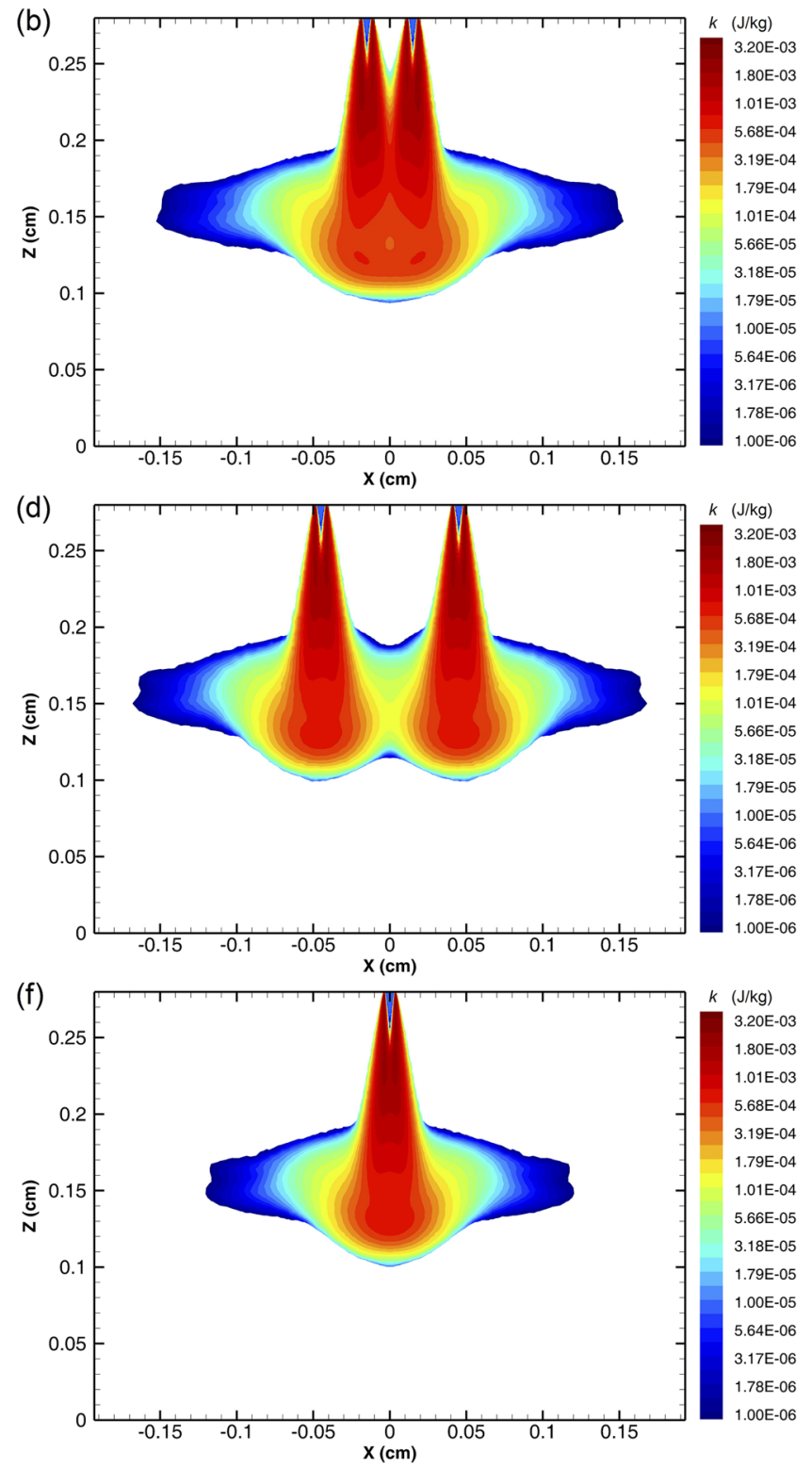

FIG. 6. Distributions of $k$ for cases 11-15 [(a)-(e)]. Those for the single plume counterpart (cases 18) are also plotted (f) for reference. 
plotted for reference. These indicate that mixing in the stems of two plumes is considerably affected by the source separation. Specifically, when two plumes are close enough, e.g., in cases 11 and 12, the plumes would significantly interact and entrain with each other, which implies that less ambient fluids would be entrained, leading to a reduced decay rate of their buoyancy flux. On the other hand, two plumes would only touch each other but not be merged if they are separate to some degree, e.g., in cases 13 and 14. In particular, under such circumstances, the rebound from the plume cap regions could create a noteworthy convection in the neutrally buoyant layer and

(a)
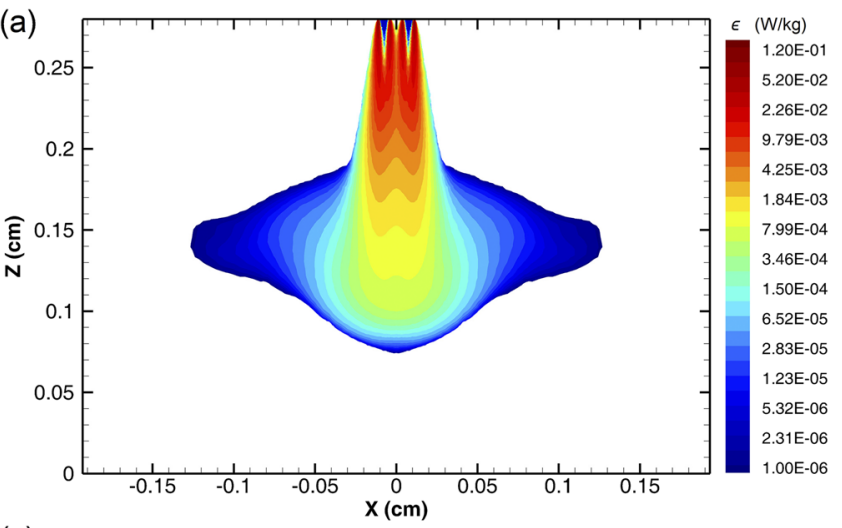

(c)
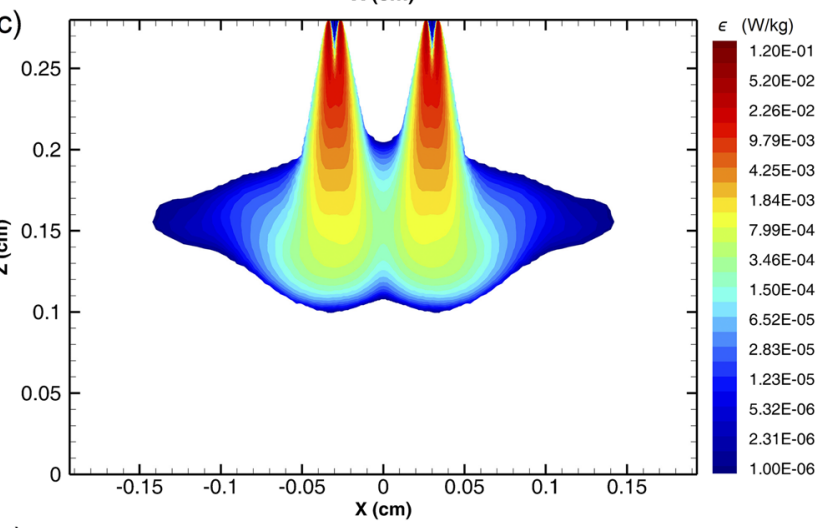

(e)

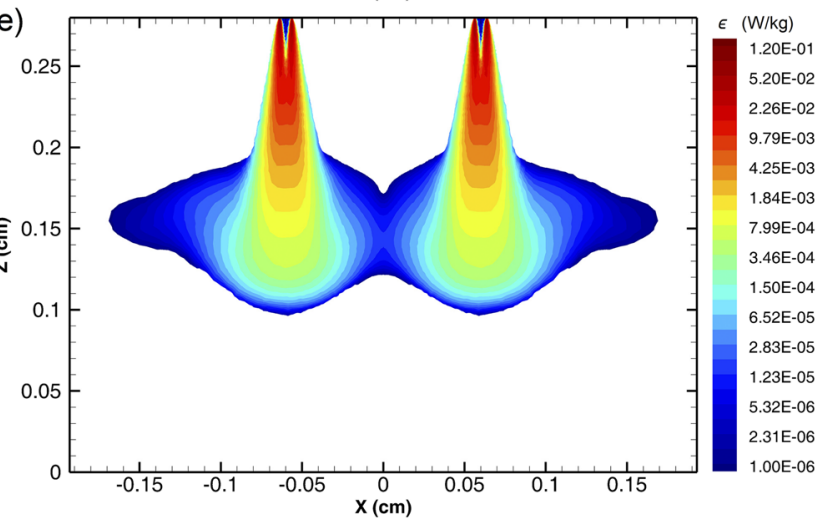

enhance the mixing here. Additionally, when the source separation is quite large, e.g., in cases 15 and 18, the two plumes propagate independently, and the entrainment in each plume stem is unaffected by the other one.

In addition, the radial profile of the normalized vertical velocity $w_{\text {rescale }}$, which is obtained by dividing it by the magnitude of local maximum down flow, in the neutrally buoyant layer is shown in Fig. 4 for cases 11-14, compared with that of the physical experiments. It suggests that the numerical predictions agree well with the experimental observations. Both of these suggest that $w_{\text {rescale }}$ displays (b)
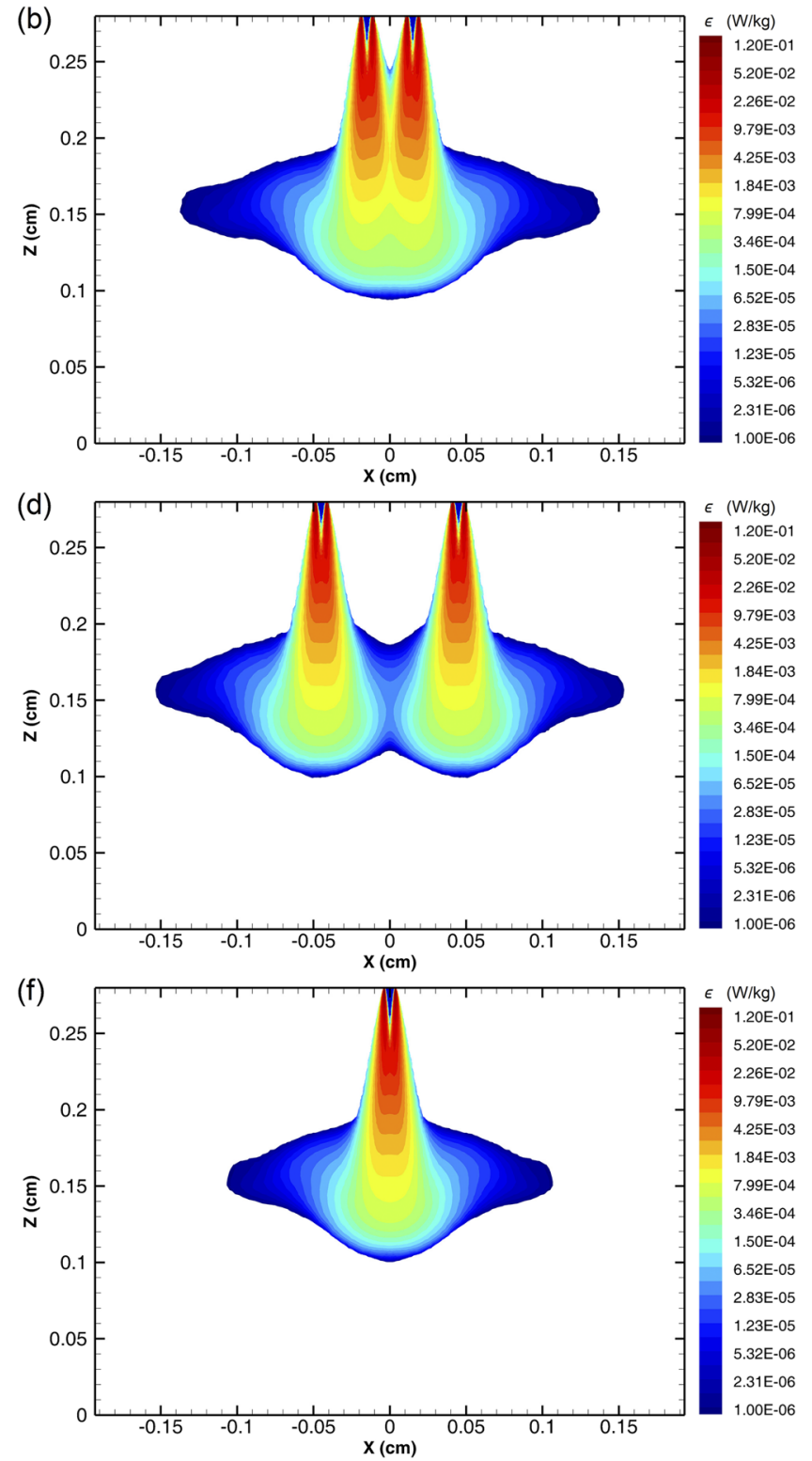

FIG. 7. Distributions of $\varepsilon$ for cases 11-15 [(a)-(e)]. Those for the single plume counterpart (cases 18) are also plotted (f) for reference. 
a Gaussian distribution in each stem, and the whole structure of the radial profiles changes from one peak to two separate peaks along with the increase in source separation.

\section{B. Maximum plume rise height scaling}

Both the results of the numerical and physical experiments suggest that there is a strong correlation between the maximum rise height of two coalescing plumes and the source separation. In order to provide an estimate of that scaling, the ratio to that of the single plume counterpart is investigated since a single plume can be
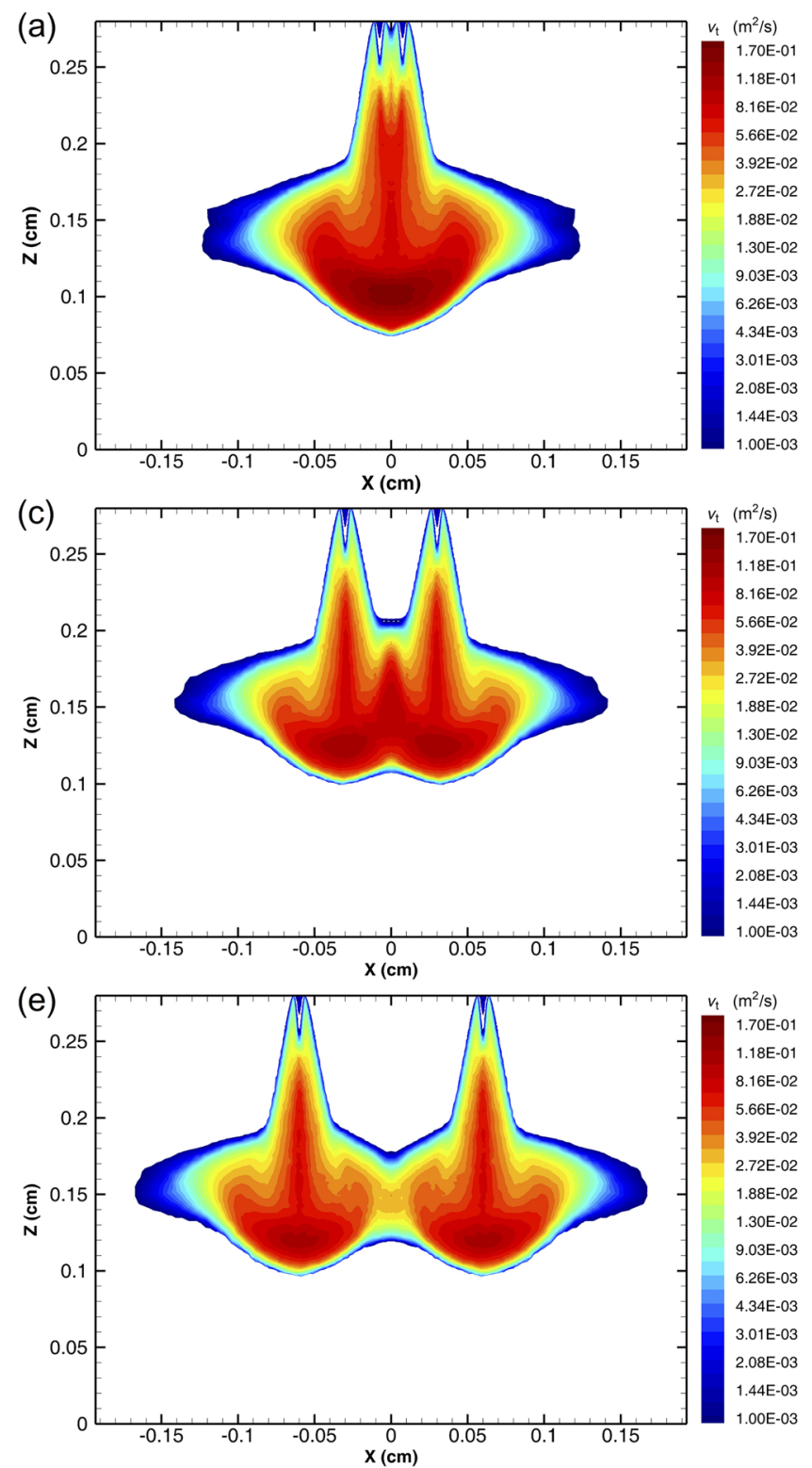

well described by the MTT model. Specifically, the maximum rise height of two plumes $Z_{\max }^{\prime}$ is transformed into $\zeta Z_{\max }$, where $Z_{\max }$ is the maximum rise height of the single plume counterpart and $\zeta$ is defined as an "amplification coefficient." The numerical and experimental results ${ }^{62}$ for all cases are listed in Table II and shown in Fig. 5, and these data indicate that the model predictions are in good agreement with the observations. Specifically, the maximum rise heights of the single plume counterparts for the numerical model were estimated by the MTT model, i.e., $Z_{\max }=3.76 B_{\text {exit }}{ }^{1 / 4} N^{-3 / 4}$. It should be noted that the classical coefficient (i.e., 3.76) is employed here since it makes a satisfactory prediction for case 16 while the

FIG. 8. Distribution of $v_{t}$ for cases 11-15 [(a)-(e)]. That for the single plume counterpart (case 18) is also plotted (f) for reference. 
integral model for forced plumes ${ }^{18}$ tends to cause an underestimate. It is noteworthy that such an underestimate may be partly due to the variability of the entrainment coefficient and the omission of the rebounding after overshooting, which leads to the lateral spreading in the neutrally buoyant layer and hence reduces the entrainment of ambient fluids.

Furthermore, since $N$ is treated as a constant in the simulations, it is reasonable to assume that $\zeta$ is a function of $L$ and $R i$ only and they are independent, i.e., $\zeta=f(L, R i)=f_{1}(L) f_{2}(R i)$. Using $B_{\text {exit }}{ }^{1 / 4} N^{-3 / 4}$ to obtain dimensionless length, the dimensionless source separation can be defined as

$$
L^{*}=\frac{L}{B_{\text {exit }}{ }^{1 / 4} N^{-3 / 4}}
$$

and directly yields $\zeta=f_{1}^{*}\left(L^{*}\right) f_{2}^{*}(R i)$.

Meanwhile, it can be noticed that the interaction between two plumes could be neglected when the source separation is large enough, which means $\lim _{L^{*} \rightarrow \infty} f_{1}^{*}\left(L^{*}\right)=1$. On the other hand, when the nozzle distance is zero, which implies that the two plumes are initially completely merged, the flow is equivalent to a single plume whose source buoyancy flux is double, i.e., $\lim _{L^{*} \rightarrow 0} f_{1}^{*}\left(L^{*}\right)=\sqrt[4]{2}$. Besides, if the source Richardson number were small enough, the two plumes would have the properties of pure jets, i.e., $\lim _{R i \rightarrow 0} f_{2}^{*}(R i)$ $=1.32$ where the entrainment coefficient for the jets with "top-hat" profiles is considered as 0.075 at the suggestion of List. ${ }^{73}$ Conversely, the flow regime will tend toward lazy plumes when $R i$ is sufficiently large, which implies that $\lim _{R i \rightarrow \infty} f_{2}^{*}(R i)=1$ is expected for such a circumstance.

According to the boundary conditions above, an empirical parameterization may be proposed as

$$
\zeta=f_{1}^{*}\left(L^{*}\right) f_{2}^{*}(R i)=\left[c_{1} \exp \left(k_{1} L^{* k_{2}}\right)+c_{2}\right]\left[c_{3} \exp \left(k_{3} R i^{k_{4}}\right)+c_{4}\right],
$$

where $c_{1}=\sqrt[4]{2}-1, c_{2}=c_{4}=1, c_{3}=0.32$, and all $k_{i}$ are undetermined coefficients. Then, determining all $k_{i}$ by a nonlinear fitting, the parameterization can be expressed as

$$
\zeta=\left[0.19 \exp \left(-1.91 L^{* 1.61}\right)+1\right]\left[0.32 \exp \left(-4.56 R i^{0.29}\right)+1\right],
$$

where the R-squared is 0.9996 . This empirical equation implies that if the maximum rise height of two coalescing submarine hydrothermal plumes in a typical ocean environment $\left(L^{*} \sim 0.5, R i \sim 1\right)^{40}$ is estimated by a single plume model with an equivalent total flow rate, the relative difference may be less than $8 \%$. Conversely, neglecting of an adjacent hydrothermal vent could underestimate this scaling by $10 \%$.

\section{Turbulence structure}

Since the two-equation $k-\varepsilon$ model has been employed to describe the turbulent effect, plume turbulence can be characterized by the turbulent kinetic energy $(k)$, the turbulence dissipation rate $(\varepsilon)$, and the kinematic turbulent viscosity $\left(v_{t}=\mu_{t} / \rho\right)$, with $\rho$ being the fluid density. Specifically, $k$ and $\varepsilon$ are obtained by solving their respective transport equations, and their variations, along with the increase in source separation, are shown in
Figs. 6 and 7 , respectively. The results suggest that the distributions of both $k$ and $\varepsilon$ are similar to that of the velocity magnitude, which shows a mushroom-like spatial distribution and reaches its maximum value in the near vent region and then decreases outwards significantly. Also, as expected, the maximum values of both $k$ and $\varepsilon$ increase monotonically with the increase in source buoyancy flux and have a negative correlation with the source separation in general.

The contours of $v_{t}$ for the cases with different source separations are plotted in Fig. 8. It indicates that the distribution of the turbulent viscosity is significantly different from that of both $k$ and $\varepsilon$. Specifically, $v_{t}$ attains its maximum in the plume cap region and would be enhanced near the neutrally buoyant layer because of rebounding and lateral spreading if the source separation is appropriate. Both of these facts, which have also been reported in related studies, ${ }^{31,40,62}$ imply that there is an important turbulent mixing process in plume cap regions, and there could be another noteworthy one in the interaction area of two plumes.

In order to provide an estimation of the relationship between the maximum turbulent viscosities of two plumes in different cases, a dimensionless turbulent viscosity is proposed as

$$
v_{t}^{*}=\frac{v_{t}}{B_{\text {exit }}{ }^{1 / 2} N^{-1 / 2}} .
$$

Normalized by the maximum value of $v_{t}{ }^{*}$ of case 8 for comparison, the normalized maximum turbulent viscosities $v_{m \text { (Case } i \text { ) }}^{*}$ $=\max \left(v_{t}^{*}\right)_{(\text {Case } i)} / \max \left(v_{t}^{*}\right)_{(\text {Case } 8)}$ for all cases are shown in Fig. 9 . It should be noted that $v_{m}^{*}$ is assumed as a function of $L^{*}$ only since $B_{\text {exit }}$ has been used to obtain the dimensionless variables and the effect of $R i$ on the flow in the plume cap regions can be limited (and will be discussed in Sec. III D).

The variation tendency suggests that $v_{m}^{*}$ changes nonmonotonically along with the increase in source separations and includes two enhancements. Specifically, the peak at $\sim 0.2$ may be due to the transported turbulence from the touching region where both the interaction and the distortion of two plumes are significant. Meanwhile, the enhancement at $\sim 2.1$ can largely be attributed to the

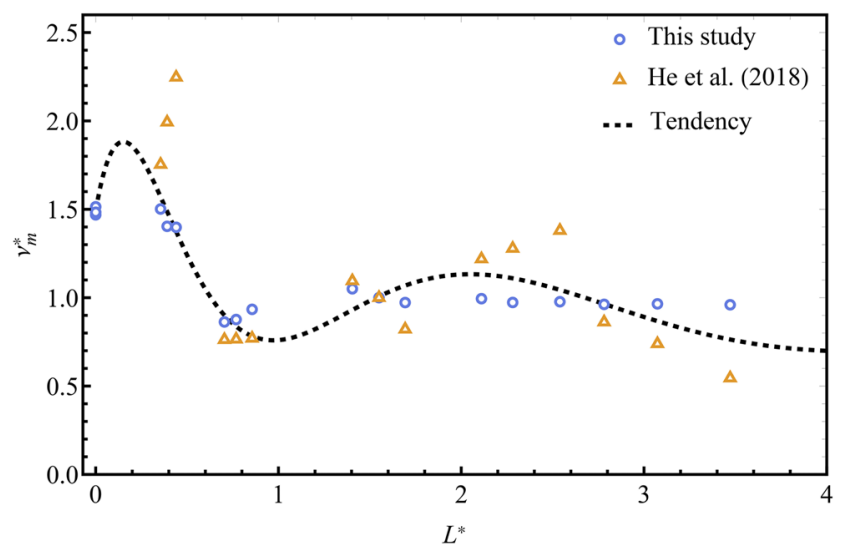

FIG. 9. Variation of $v_{m}^{*}$ along with $L^{*}$ for all cases. The dotted line indicates its tendency. 
(a)

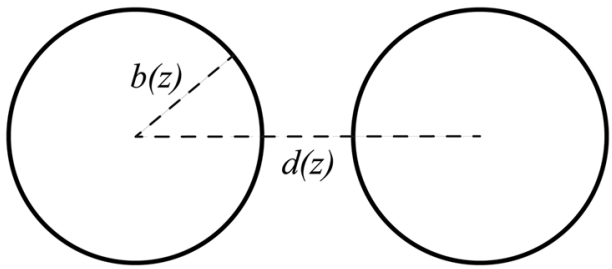

(b)

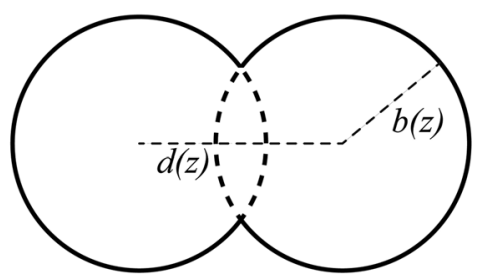

(c)

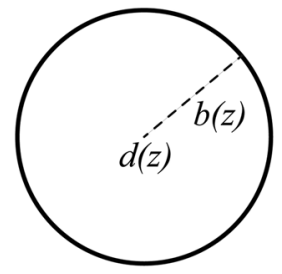

FIG. 10. Schematic of horizontal slices of (a) two independent plumes, (b) two touching plumes, and (c) a merged plume with "top-hat" profiles.

lateral convection around the neutrally buoyant layer between two plumes since a significant mixing region can be observed there, and such a spreading is caused by the rebound after overshooting.

\section{Entrainment coefficient}

In the MTT model, the entrainment coefficient $\alpha$ is defined to provide a feasible way to close the conservation equations. Since it represents the ratio of the horizontal entrainment velocity to the mean vertical velocity, its value depends on the definition of the plume horizontal length scale and the radial profile of axial velocity. ${ }^{74}$ For consistency, all values of $\alpha$ involved in this study have been converted to values with respect to a "top-hat" profile according to the conservation of volume and momentum flux. In particular, at the suggestion of Cenedese and Linden, ${ }^{65}$ the shape of two plumes with "top-hat" profiles is assumed to be that depicted in Fig. 10, where $b(z)$ is the plume horizontal length scale and $d(z)$ is the separation between two centerlines determined by the local peak value of the vertical velocity, and $z$ is a downward vertical coordinate whose origin is located at the center of inlet plane (i.e., $z=0.28-Z$ ).

By definition, the rate of change of the plume volume flux can be expressed as

$$
\frac{d}{d z} Q(z)=\alpha P(z) \frac{Q(z)}{A(z)},
$$

where $Q(z)$ is the total volume flux and $P(z)$ and $A(z)$ are the perimeter and the horizontal areas of the two plumes with "top-hat" profiles, respectively. Accordingly, $\alpha$ for all cases of the experimental group are given in Fig. 11 after being processed by a low-pass filter. The results imply that the classical entrainment assumption (i.e., $\alpha$ is always constant) is only applicable to the plume stem below $\sim 0.5 Z_{\max }^{\prime}$. Above that level, the convection in the neutrally buoyant layer dominates the flow, and such lateral spreading would lead to a rapid decrease in the entrainment coefficient.

Moreover, those profiles suggest that, in the near vent regions, there is a strong positive correlation between $\alpha$ and the source
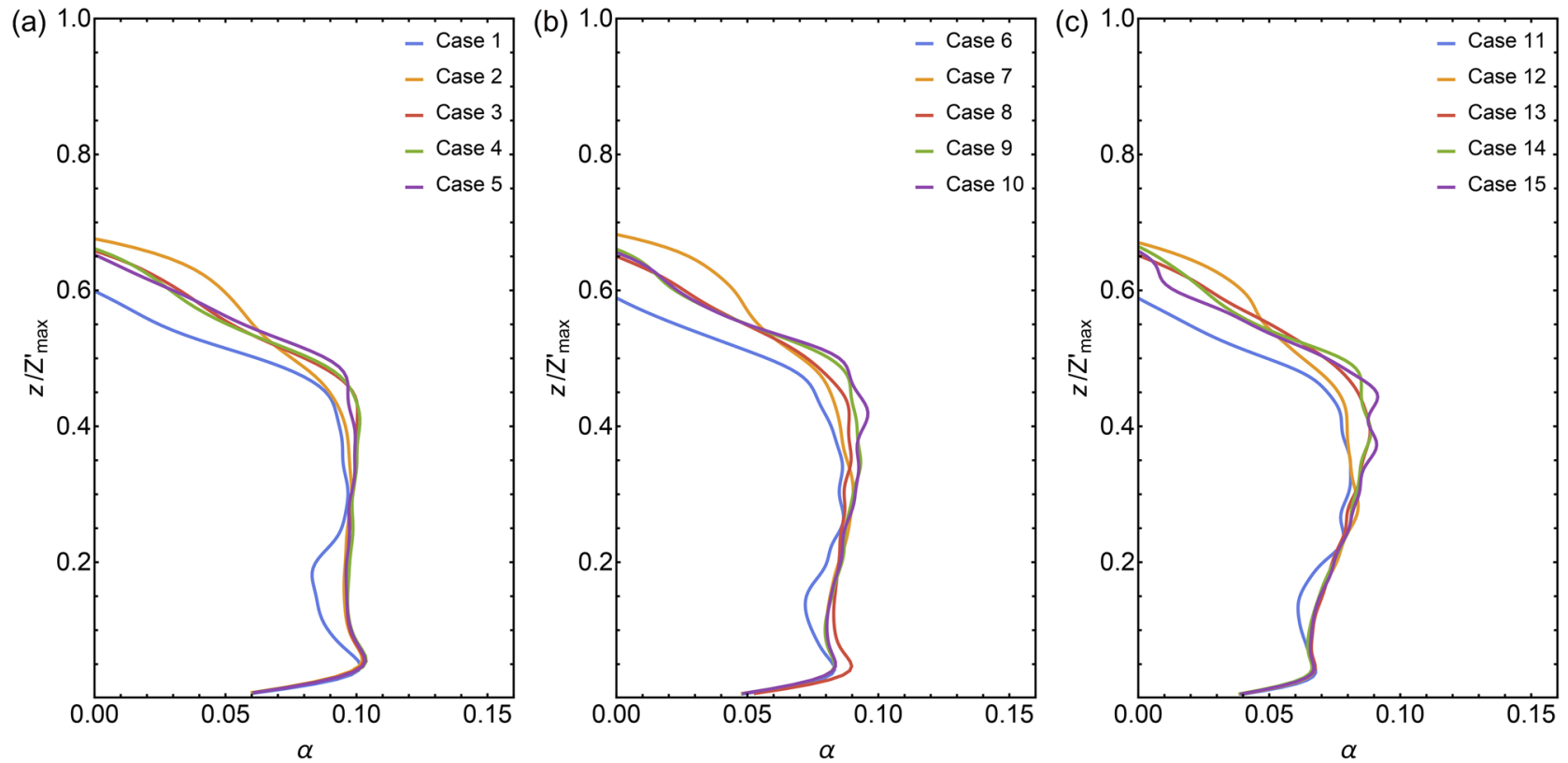

FIG. 11. Vertical profiles of the entrainment coefficient $\alpha$ for (a) cases 1-5, (b) cases 6-10, and (c) cases 11-15 

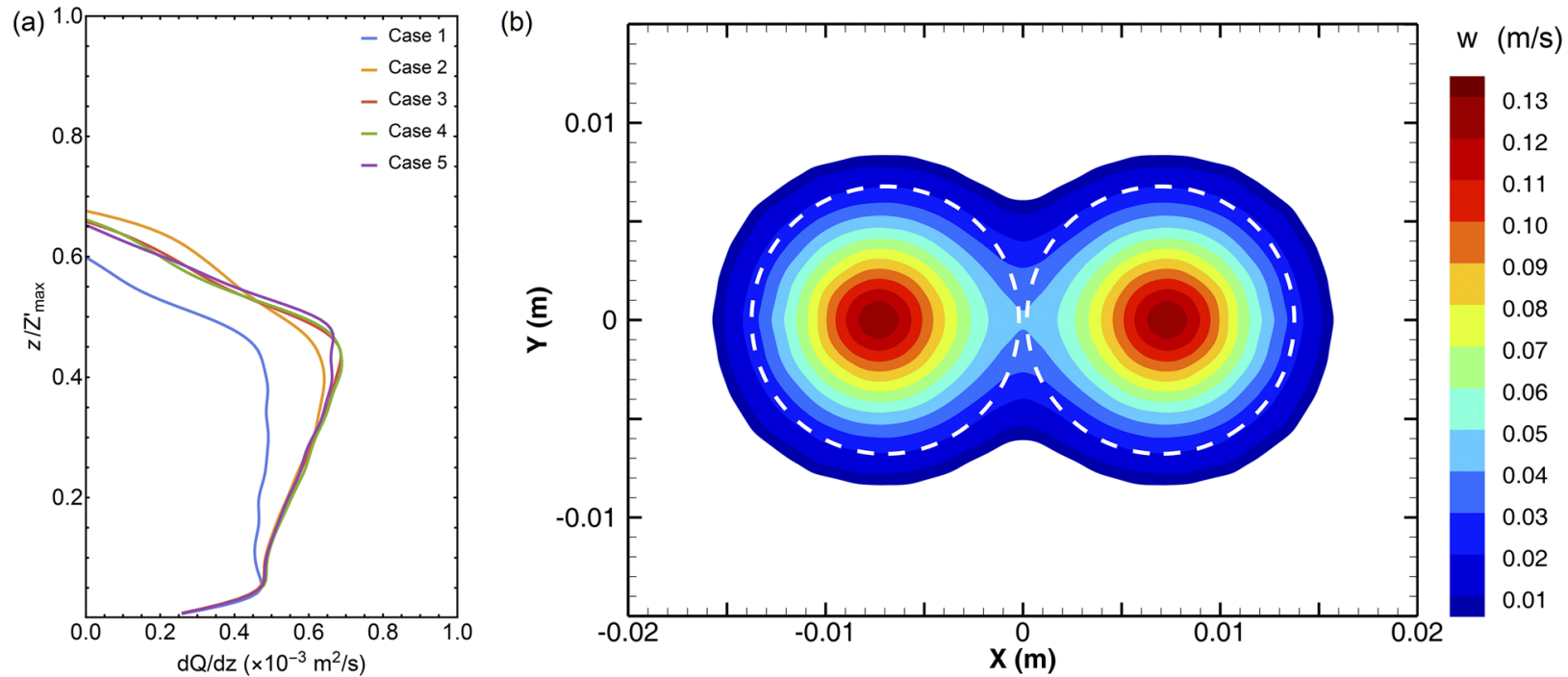

FIG. 12. Vertical profiles of $d Q / d z$ for cases $1-5$ (a) and the horizontal profile of the vertical velocity $w$ at $0.15 Z^{\prime}{ }_{\text {max }}$ for case 1 (b). The dashed white lines indicate the boundary of the plumes with "top-hat" profiles.

Richardson number. Furthermore, there are noteworthy slopes indicating a transition between flow regimes in those cases with a low $R i$ [Fig. 11(c)]. This fact implies that forced plumes may have a tendency to regulate themselves to plume-like flows.

It is noteworthy that there are remarkable "dips" around $0.15 Z_{\max }^{\prime}$ in Fig. 11. To investigate the implication of these dips, the vertical profiles of the change rate of the volume flux for cases 1-5 are shown in Fig. 12(a), while the horizontal profile of the vertical velocity $w$ at $0.15 Z_{\max }^{\prime}$ for case 1 is shown in Fig. 12(b). The vertical profiles of $d Q / d z$ suggest that the entrainment of ambient fluids will decrease when two plumes touch each other. The decrease implies that mutual entrainment between two plumes would play an (a)

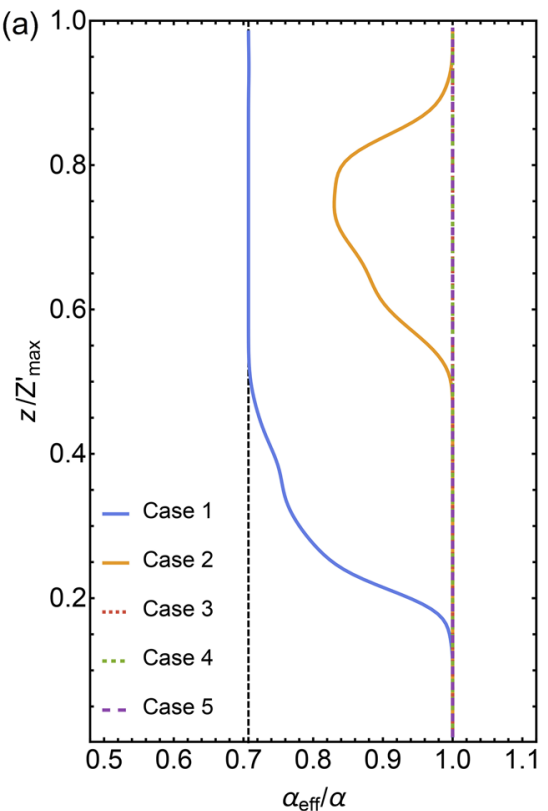

(b)

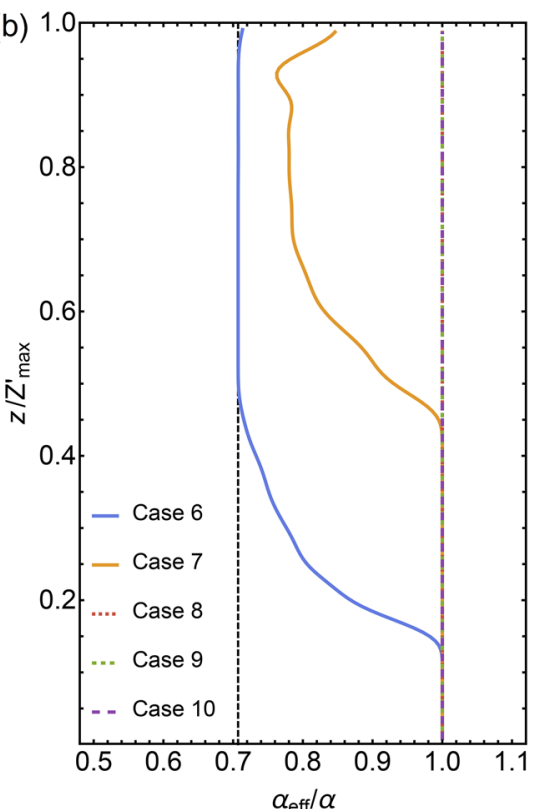

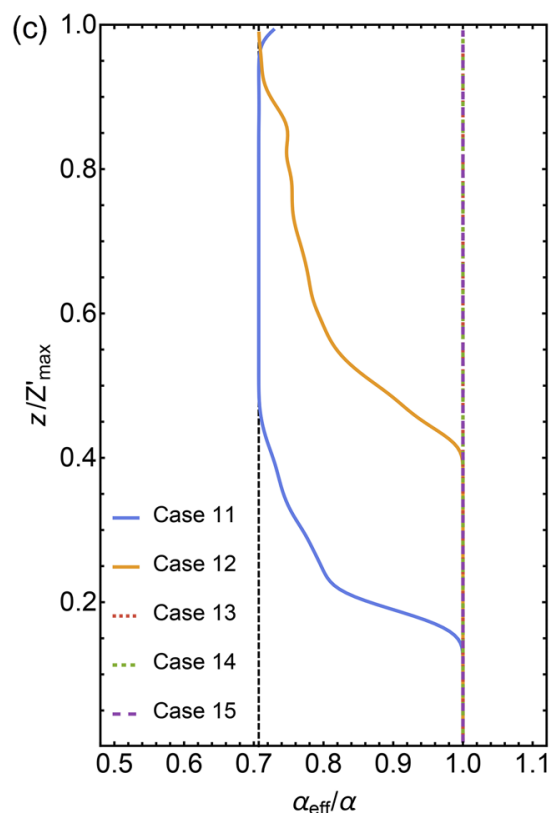

FIG. 13. The dependence of the ratio of the effective entrainment coefficient to the entrainment coefficient for (a) cases 1-5, (b) cases 6-10, and (c) cases 11-15 with depth The black dashed line indicates the theoretical value of two merged plumes, i.e., $2^{-1 / 2}$. 
important role when the interaction of the two plumes is significant. Moreover, the superposition of two plumes will lead to asymmetric horizontal decay rates of vertical velocity and then result in distortion of plume boundaries, like those shown in Fig. 12(b). The distortion, however, can potentially enhance the entrainment near the vertical plane of symmetry since a higher vertical velocity could be maintained here. In this sense, the distortion may be regarded as a modification to the decrease in entrainment.

Furthermore, the "effective" entrainment coefficient $\alpha_{\text {eff }}$ proposed by Cenedese and Linden ${ }^{65}$ is introduced to parameterize the combined behavior of two plumes. Specifically, this parameter is defined as the value of $\alpha$ needed for two isolated plumes to maintain both the same total volume and momentum flux as that of two touching plumes. Since the touching height of two plumes $z_{T}$ is defined as the height where the plume horizontal length scale is equal to half the separation between two centerlines, i.e., $b\left(z_{T}\right)=d\left(z_{T}\right) / 2$, the definition implies that $\alpha_{\text {eff }}$ will begin to decrease at the touching height. Hence, the profiles of $\alpha_{\text {eff }}$ shown in Fig. 13 suggest that, as discussed in Sec. III A, the two plumes with a small source separation would be merged, while those with a large source separation could remain independent and those with a moderate nozzle separation (e.g., cases 2, 7, and 12) constitute a transitional state.

\section{CONCLUSIONS}

The present study investigates the hydrodynamics of two coalescing turbulent forced plumes in linearly stratified fluids using a numerical approach and compares the results with a series of physical experiments. Typical structures of both flow and turbulence fields of two forced plumes are obtained to analyze vertical velocity profiles, maximum plume rise heights, turbulent viscosity, and entrainment coefficient quantitatively.

The results suggest that the radial profile of vertical velocity in the stems of two plumes can be approximated by a mixture of two Gaussian distributions and is closely related to the source separation. Specifically, if the nozzle distance is short enough, two plumes would be merged and result in less entrainment of the ambient fluid. When the source separation is moderate, two plumes may only touch each other and cause a notable mixing near the neutrally buoyant layer. Once two plumes are quite separate, they tend to propagate independently.

Because of less entrainment of ambient fluid, the maximum rise height of two coalescing forced plumes is considerably greater than that of their single plume counterpart. An empirical parameterization as a function of the dimensionless source separation and the source Richardson number has been proposed to predict this scaling. This prediction agrees well with experimental observations.

The maximum values of both the turbulent kinetic energy and the turbulence dissipation rate of two turbulent plumes increase monotonically with the increase in source buoyancy flux and have a notable negative correlation with the source separation. However, the maximum turbulent viscosity, which attains its maximum in the plume cap region, includes two enhancements along with the increase in source separation. The extra turbulence in the plume cap region for those cases with a very small source separation may be transported from the touching region where both plume interactions and distortions are significant. The enhancement for those cases with a moderate source separation, however, can be largely attributed to lateral spreading and strong mixing around the neutrally buoyant layer between two plumes caused by the rebound after overshooting.

In the near vent regions, the entrainment coefficient of two forced plumes with "top-hat" profiles has a positive relationship with the source Richardson number. Meanwhile, in plume stems below $\sim 0.5 Z_{\text {max }}^{\prime}$, the entrainment coefficient remains relatively constant for those cases with a high source Richardson number, and it shows an increasing tendency with the decrease in the source Richardson number. Above that level, the flow is dominated by the lateral spreading around the neutrally buoyant layer, and hence the entrainment coefficient decreases to zero rapidly. Furthermore, the entrainment coefficient will decrease in plume touching regions due to mutual entrainment. The superposition of plumes can lead to a lower horizontal decay rate of vertical velocity between the two plumes and then produce distortion of plume cross sections.

\section{ACKNOWLEDGMENTS}

This work was financially supported by the National Natural Science Foundation of China (Grant No. 11672267) and Fundamental Research Funds for the Central Universities (Grant No. 2017XZZX001-02A). This work was supported by HPC Center of ZJU (Zhoushan campus). Yingzhong Lou would like to thank Liang Zhao at Zhejiang University for fruitful discussions. The authors gratefully acknowledge the constructive suggestions offered by the anonymous referees.

\section{APPENDIX A: GOVERNING EQUATIONS}

The model developed in this study is performed using the finite-volume CFD software package ANSYS Fluent. The main governing equations are as follows.

The Reynolds averaged model is adopted in this study. Dropping the overbar on the mean variables, the ensemble-averaged continuity and momentum equations for the species transport model can be written in Cartesian tensor form as

$$
\begin{gathered}
\frac{\partial}{\partial t}\left(\rho Y_{i}\right)+\frac{\partial}{\partial x_{k}}\left(\rho u_{k} Y_{i}\right)=-\frac{\partial}{\partial x_{k}} J_{i, k}, \\
\frac{\partial}{\partial t}\left(\rho u_{i}\right)+\frac{\partial}{\partial x_{j}}\left(\rho u_{i} u_{j}\right)=-\frac{\partial p}{\partial x_{i}}+\frac{\partial \tau_{i j}}{\partial x_{j}}+\frac{\partial}{\partial x_{j}}\left(-\overline{\rho{u^{\prime}}_{i} u_{j}^{\prime}}\right)+\rho g_{i},
\end{gathered}
$$

where $u_{i}$ and $u_{i}^{\prime}$ are the mean and fluctuating velocity components. $Y_{i}$ is the local mass fraction of species $i . \rho=1 /\left(\sum Y_{i} / \rho_{i}\right)$ is the mixture density. $\vec{J}_{i}$ is the diffusion flux of species $i$, computed from $\vec{J}_{i}=-\left(\rho D_{i, m}+\mu_{t} / S c_{t}\right) \nabla Y_{i}$, where $D_{i, m}$ is the mass diffusion coefficient for species $i$ in the mixture; $S c_{t}$ is the turbulent Schmidt number; $\mu_{t}$ is the turbulent viscosity. The mass diffusion in turbulent flow is considered as the primary phase interaction in the model. As the salinity involved in the model is relatively low, the diffusion coefficient for species is determined by the constant dilute approximation method, and the scaling is approximated by the diffusion coefficient for sodium chloride suggested by Haynes et al. ${ }^{75} \tau_{i j}$ is the stress tensor given by $\tau_{i j}=\mu\left(\frac{\partial u_{i}}{\partial x_{j}}+\frac{\partial u_{j}}{\partial x_{i}}-\frac{2}{3} \delta_{i j} \frac{\partial u_{l}}{\partial x_{l}}\right)$ where $\mu$ is the molecular viscosity $\left(1.003 \times 10^{-3} \mathrm{~kg} \mathrm{~m}^{-1} \mathrm{~s}^{-1}\right.$ is used here $) .-\rho \overline{u^{\prime}{ }_{i}{ }^{\prime}}{ }_{j}$ are 
Reynolds stresses, which can be modeled in Cartesian tensor form using the Boussinesq hypothesis as

$$
-\rho \overline{u_{i}^{\prime} u_{j}^{\prime} j}=\mu_{t}\left(\frac{\partial u_{i}}{\partial x_{j}}+\frac{\partial u_{j}}{\partial x_{i}}\right)-\frac{2}{3}\left(\rho k+\mu_{t} \frac{\partial u_{k}}{\partial x_{k}}\right) \delta_{i j},
$$

where $k$ is the turbulent kinetic energy and $\delta_{i j}$ is the Kronecker delta.

The turbulent viscosity $\mu_{t}$ is given by

$$
\mu_{t}=\rho C_{\mu} \frac{k^{2}}{\varepsilon}
$$

where $\varepsilon$ is the turbulence dissipation rate and $C_{\mu}$ is the coefficient described below.

The realizable $k-\varepsilon$ model $^{76}$ is employed in this study. The transport equations for $k$ and $\varepsilon$ in the model are

$$
\frac{\partial}{\partial t}(\rho k)+\frac{\partial}{\partial x_{j}}\left(\rho k u_{j}\right)=\frac{\partial}{\partial x_{j}}\left[\left(\mu+\frac{\mu_{t}}{\sigma_{k}}\right) \frac{\partial k}{\partial x_{j}}\right]+G_{k}+G_{b}-\rho \varepsilon
$$

and

$$
\begin{aligned}
\frac{\partial(\rho \varepsilon)}{\partial t}+\frac{\partial\left(\rho \varepsilon u_{j}\right)}{\partial x_{j}}= & \frac{\partial}{\partial x_{j}}\left[\left(\mu+\frac{\mu_{t}}{\sigma_{\varepsilon}}\right) \frac{\partial \varepsilon}{\partial x_{j}}\right]+\rho C_{1} S \varepsilon \\
& -\rho C_{2} \frac{\varepsilon^{2}}{k+\sqrt{\varepsilon \mu / \rho}}+C_{1 \varepsilon} \frac{\varepsilon}{k} C_{3 \varepsilon} G_{b},
\end{aligned}
$$

where $G_{k}=-\rho \overline{u_{i}^{\prime} u^{\prime} j} \frac{\partial u_{j}}{\partial x_{i}}=\mu_{t} S^{2}$ represents the generation of turbulent kinetic energy due to the mean velocity gradients, where $S=\sqrt{2 S_{i j} S_{i j}}$ is the modulus of the mean rate-of-strain tensor and $S_{i j} \equiv \frac{1}{2}\left(\frac{\partial u_{i}}{\partial x_{j}}+\frac{\partial u_{j}}{\partial x_{i}}\right)$ is the mean strain rate. $G_{b}=\beta g_{i} \frac{\mu_{t}}{P r_{t}} \frac{\partial T}{\partial x_{i}}$ is the generation of turbulent kinetic energy due to buoyancy, with $P r_{t}$ $=0.85$ as the turbulent Prandtl number for energy and $\beta=-\frac{1}{\rho}\left(\frac{\partial \rho}{\partial T}\right)_{P}$ as the coefficient of thermal expansion. $C_{3 \varepsilon}=\tanh (|w / v|)$ determines the degree to which $\varepsilon$ is affected by the buoyancy, and $w$ and $v$ are the components of the flow velocity parallel and perpendicular to the gravitational vector, respectively. $\sigma_{k}=1.0$ and $\sigma_{\varepsilon}=1.2$ are the turbulent Prandtl numbers for $k$ and $\varepsilon$, respectively. $C_{1}=\max [0.43, \eta /(\eta+5)], C_{2}=1.9, C_{1 \varepsilon}=1.44$ are model constants with $\eta=S k / \varepsilon$. by

In the realizable $k-\varepsilon$ model, $C_{\mu}$ is no longer constant and given

$$
C_{\mu}=\frac{1}{A_{0}+A_{S} \frac{k U^{*}}{\varepsilon}},
$$

where $U^{*}=\sqrt{S_{i j} S_{i j}+\Omega_{i j} \Omega_{i j}}$ where $\Omega_{i j}=\bar{\Omega}_{i j}-\varepsilon_{i j k} \omega_{k}, \bar{\Omega}_{i j}$ $=\frac{1}{2}\left(\frac{\partial u_{i}}{\partial x_{j}}-\frac{\partial u_{j}}{\partial x_{i}}\right)$ is the mean rotation-rate tensor, and $\omega_{k}$ is the angular velocity; $A_{0}=4.04, A_{S}=\sqrt{6} \cos \phi$ are model constants with $\phi=\frac{1}{3} \arccos (\sqrt{6} \omega)$ and $\omega=\frac{s_{i j} S_{j l} S_{l i}}{(s / \sqrt{2})^{3}}$.

\section{APPENDIX B: NUMERICAL METHODS}

The governing equations were numerically solved by ANSYS Fluent. The Green-Gauss Node-Based Gradient Evaluation method was used to compute the gradients since this method can be more accurate than the cell-based methods on unstructured meshes. The Third-Order MUSCL (Monotone Upstream-centered Schemes for
Conservation Laws) scheme was selected as the spatial discretization method for the momentum, turbulence, and mass fraction equations. The PRESTO! (PREssure STaggering Option) scheme was employed as the discretization method for the pressure. The coupled algorithm was used for pressure-velocity coupling, and the secondorder Implicit scheme was applied as the temporal discretization method. All simulations were run in parallel on the Linux cluster maintained by the HPC Center of Zhejiang University (Zhoushan Campus).

\section{REFERENCES}

${ }^{1}$ R. S. J. Sparks, M. I. Bursik, S. N. Carey, J. Gilbert, L. S. Glaze, H. Sigurdsson, and A. W. Woods, Volcanic Plumes (John Wiley \& Sons, Chichester, 1997).

${ }^{2}$ S. Kimura, P. R. Holland, A. Jenkins, and M. Piggott, "The effect of meltwater plumes on the melting of a vertical glacier face," J. Phys. Oceanogr. 44, 3099-3117 (2014).

${ }^{3}$ D. A. Slater, P. W. Nienow, T. R. Cowton, D. N. Goldberg, and A. J. Sole, "Effect of near-terminus subglacial hydrology on tidewater glacier submarine melt rates," Geophys. Res. Lett. 42, 2861-2868, https://doi.org/10.1002/2014gl062494 (2015).

${ }^{4}$ A. C. Lai and J. H. Lee, "Dynamic interaction of multiple buoyant jets," J. Fluid Mech. 708, 539-575 (2012).

${ }^{5}$ J. H. W. Lee, "Mixing of multiple buoyant jets," J. Hydraul. Eng. 138, 1008-1021 (2012).

${ }^{6}$ J. E. Lupton, "Hydrothermal plumes: Near and far field," in Seafloor Hydrothermal Systems: Physical, Chemical, Biological, and Geological Interactions, Volume 91 of the Geophysical Monograph Series, edited by S. E. Humphris, R. A. Zierenberg, L. S. Mullineaux, and R. E. Thomson (American Geophysical Union, 1995), pp. 317-346.

${ }^{7}$ E. T. Baker, "Hydrothermal plumes," in Encyclopedia of Marine Geosciences, Encyclopedia of Marine Geosciences, edited by J. Harff, M. Meschede, S. Petersen, and J. Thiede (Springer, 2016), pp. 335-339.

${ }^{8}$ J. Francheteau, H. D. Needham, P. Choukroune, T. Juteau, M. Seguret, R. D. Ballard, P. J. Fox, W. Normark, A. Carranza, and D. Cordoba, "Massive deep-sea sulphide ore deposits discovered on the east pacific rise," Nature 277, 523-528 (1979).

${ }^{9}$ Y. Wang, X. Han, S. Petersen, X. Jin, Z. Qiu, and J. Zhu, "Mineralogy and geochemistry of hydrothermal precipitates from Kairei hydrothermal field, Central Indian Ridge," Mar. Geol. 354, 69-80 (2014).

${ }^{10}$ Y. Wang, X. Han, S. Petersen, M. Frische, Z. Qiu, H. Li, H. Li, Z. Wu, and R. Cui, "Mineralogy and trace element geochemistry of sulfide minerals from the Wocan hydrothermal field on the slow-spreading Carlsberg Ridge, Indian Ocean," Ore Geol. Rev. 84, 1-19 (2017).

${ }^{11}$ P. Lam, J. P. Cowen, B. N. Popp, and R. D. Jones, "Microbial ammonia oxidation and enhanced nitrogen cycling in the endeavour hydrothermal plume," Geochim. Cosmochim. Acta 72, 2268-2286 (2008).

${ }^{12}$ G. J. Dick and B. M. Tebo, "Microbial diversity and biogeochemistry of the Guaymas Basin deep-sea hydrothermal plume," Environ. Microbiol. 12, 1334-1347 (2010).

${ }^{13}$ R. A. Lesniewski, S. Jain, K. Anantharaman, P. D. Schloss, and G. J. Dick, "The metatranscriptome of a deep-sea hydrothermal plume is dominated by water column methanotrophs and lithotrophs," ISME J. 6, 2257-2268 (2012).

${ }^{14} \mathrm{D}$. Kadko, "An assessment of the effect of chemical scavenging within submarine hydrothermal plumes upon ocean geochemistry," Earth Planet. Sci. Lett. 120, 361-374 (1993).

${ }^{15}$ J. A. Breier, B. M. Toner, S. C. Fakra, M. A. Marcus, S. N. White, A. M. Thurnherr, and C. R. German, "Sulfur, sulfides, oxides and organic matter aggregated in submarine hydrothermal plumes at $9^{\circ} 50^{\prime} \mathrm{N}$ East Pacific rise," Geochim. Cosmochim. Acta 88, 216-236 (2012).

${ }^{16}$ J. A. Resing, P. N. Sedwick, C. R. German, W. J. Jenkins, J. W. Moffett, B. M. Sohst, and A. Tagliabue, "Basin-scale transport of hydrothermal dissolved metals across the south pacific ocean," Nature 523, 200-203 (2015).

${ }^{17}$ B. R. Morton, G. Taylor, and J. S. Turner, "Turbulent gravitational convection from maintained and instantaneous sources," Proc. R. Soc. A 234, 1-23 (1956). 
${ }^{18}$ B. R. Morton, “Forced plumes," J. Fluid Mech. 5, 151-163 (1959).

${ }^{19}$ C. S. Yih, “Turbulent buoyant plumes," Phys. Fluids 20, 1234-1237 (1977).

${ }^{20} \mathrm{~J}$. W. Lavelle, "The initial rise of a hydrothermal plume from a line segment source-Results from a three-dimensional numerical model," Geophys. Res. Lett. 22, 159-162, https://doi.org/10.1029/94gl01463 (1995).

${ }^{21}$ X. Zhou, K. H. Luo, and J. J. R. Williams, "Large-eddy simulation of a turbulent forced plume," Eur. J. Mech. - B/Fluids 20, 233-254 (2001).

${ }^{22}$ M. V. Pham, F. Plourde, and S. D. Kim, "Three-dimensional characterization of a pure thermal plume," J. Heat Transfer 127, 624-636 (2005).

${ }^{23}$ M. V. Pham, F. Plourde, S. D. Kim, and S. Balachandar, "Large-eddy simulation of a pure thermal plume under rotating conditions," Phys. Fluids 18, 015101 (2006).

${ }^{24} \mathrm{G}$. Michaux and O. Vauquelin, "Solutions for turbulent buoyant plumes rising from circular sources," Phys. Fluids 20, 066601 (2008).

${ }^{25}$ D. Contini, A. Donateo, D. Cesari, and A. G. Robins, "Comparison of plume rise models against water tank experimental data for neutral and stable crossflows," J. Wind Eng. Ind. Aerodyn. 99, 539-553 (2011).

${ }^{26} \mathrm{~J}$. W. Lavelle, D. D. Iorio, and P. Rona, "A turbulent convection model with an observational context for a deep-sea hydrothermal plume in a time-variable cross flow," J. Geophys. Res.: Oceans 118, 6145-6160, https://doi.org/10.1002/2013jc009165 (2013).

${ }^{27}$ T. S. Richards, Q. Aubourg, and B. R. Sutherland, "Radial intrusions from turbulent plumes in uniform stratification," Phys. Fluids 26, 036602 (2014).

${ }^{28}$ A. Fabregat Tomàs, A. C. Poje, T. M. Özgökmen, and W. K. Dewar, "Dynamics of multiphase turbulent plumes with hybrid buoyancy sources in stratified environments," Phys. Fluids 28, 095109 (2016).

${ }^{29} \mathrm{~L}$. Gao and S. C. M. Yu, "Vortex ring formation in starting forced plumes with negative and positive buoyancy," Phys. Fluids 28, 113601 (2016).

${ }^{30} \mathrm{H}$. N. Mirajkar and S. Balasubramanian, "Effects of varying ambient stratification strengths on the dynamics of a turbulent buoyant plume," J. Hydraul. Eng. 143, 04017013 (2017).

${ }^{31}$ W. Zhang, Z. He, and H. Jiang, "Scaling for turbulent viscosity of buoyant plumes in stratified fluids: PIV measurement with implications for submarine hydrothermal plume turbulence," Deep Sea Res., Part I 129, 89-98 (2017).

${ }^{32}$ F. Dabbagh, F. X. Trias, A. Gorobets, and A. Oliva, "On the evolution of flow topology in turbulent Rayleigh-Bénard convection," Phys. Fluids 28, 115105 (2016).

${ }^{33}$ A. Mazzino, "Two-dimensional turbulent convection," Phys. Fluids 29, 111102 (2017).

${ }^{34}$ B. Vajipeyajula, T. Khambampati, and R. A. Handler, "Dynamics of a single buoyant plume in a FENE-P fluid," Phys. Fluids 29, 091701 (2017).

${ }^{35}$ W. Zhou and J. Chen, "Letter: Similarity model for corner roll in turbulent Rayleigh-Bénard convection," Phys. Fluids 30, 111705 (2018).

${ }^{36}$ J. Cheng, W. Cai, H. Zhang, F. Li, L. Shen, and S. Qian, "Numerical study on the dynamic process of single plume flow in thermal convection with polymers," Phys. Fluids 31, 023105 (2019).

${ }^{37}$ J. W. Lavelle, "A convection model for hydrothermal plumes in a cross flow," Technical Report ERL PMEL-102, NOAA Technical Memorandum, NTIS, 1994.

${ }^{38} \mathrm{M}$. V. Pham, F. Plourde, and K. S. Doan, "Direct and large-eddy simulations of a pure thermal plume," Phys. Fluids 19, 125103 (2007).

${ }^{39} \mathrm{Y}$. Tao, S. Rosswog, and M. Brüggen, "A simulation modeling approach to hydrothermal plumes and its comparison to analytical models," Ocean Modell. 61, 68-80 (2013).

${ }^{40} \mathrm{H}$. Jiang and J. A. Breier, "Physical controls on mixing and transport within rising submarine hydrothermal plumes: A numerical simulation study," Deep Sea Res., Part I 92, 41-55 (2014).

${ }^{41}$ Z. Zhang, Y. Guo, J. Zeng, J. Zheng, and X. Wu, "Numerical simulation of vertical buoyant wall jet discharged into a linearly stratified environment," J. Hydraul. Eng. 144, 06018009 (2018).

${ }^{42}$ C. S. Pant and A. Bhattacharya, "Evaluation of an energy consistent entrainment model for volumetrically forced jets using large eddy simulations," Phys. Fluids $\mathbf{3 0}$, 105107 (2018).

${ }^{43} \mathrm{~K}$. G. Speer and P. A. Rona, "A model of an atlantic and pacific hydrothermal plume," J. Geophys. Res.: Oceans 94, 6213-6220, https://doi.org/10.1029/ jc094ic05p06213 (1989).
${ }^{44}$ Y. Fouquet, J. L. Charlou, U. V. Stackelberg, M. Wiedicke, J. Erzinger, P. M. Herzig, and R. Muehe, "Metallogenesis in back-arc environments: The Lau Basin example," Econ. Geol. 88, 2154-2181 (1993).

${ }^{45}$ J. I. Ishibashi, J. E. Lupton, T. Yamaguchi, J. Querellou, T. Nunoura, and K. Takai, "Expedition reveals changes in lau basin hydrothermal system," Eos, Trans., Am. Geophys. Union 87, 13-17 (2006).

${ }^{46}$ V. L. Ferrini, M. K. Tivey, S. M. Carbotte, F. Martinez, and C. Roman, "Variable morphologic expression of volcanic, tectonic, and hydrothermal processes at six hydrothermal vent fields in the Lau back-arc basin," Geochem., Geophys., Geosyst. 9, Q07022, https://doi.org/10.1029/2008gc002047 (2008).

${ }^{47}$ R. B. Bornoff and M. R. Mokhtarzadeh-Dehghan, "A numerical study of interacting buoyant cooling-tower plumes," Atmos. Environ. 35, 589-598 (2001). ${ }^{48} \mathrm{~S}$. Li, A. Moradi, B. Vickers, and M. Flynn, "Cooling tower plume abatement using a coaxial plume structure,” Int. J. Heat Mass Transfer 120, 178-193 (2018).

${ }^{49}$ P. F. Linden, G. F. Lane-Serff, and D. A. Smeed, "Emptying filling boxes: The fluid mechanics of natural ventilation," J. Fluid Mech. 212, 309-335 (1990).

${ }^{50}$ P. F. Linden, "The fluid mechanics of natural ventilation," Annu. Rev. Fluid Mech. 31, 201-238 (1999).

${ }^{51}$ D. Anfossi, G. Bonino, F. Bossa, and R. Richiardone, "Plume rise from multiple sources: A new model," Atmos. Environ. 12, 1821-1826 (1978).

${ }^{52}$ M. J. Davidson, D. A. Papps, and I. R. Wood, "The behaviour of merging buoyant jets," in Recent Research Advances in the Fluid Mechanics of Turbulent Jets and Plumes, edited by P. A. Davies and M. J. V. Neves (Springer, 1994), Vol. 255, pp. $465-478$.

${ }^{53}$ P. C. Yannopoulos, "Advanced integral model for groups of interacting round turbulent buoyant jets," Environ. Fluid Mech. 10, 415-450 (2010).

${ }^{54}$ S. Alessandrini, E. Ferrero, and D. Anfossi, "A new Lagrangian method for modelling the buoyant plume rise," Atmos. Environ. 77, 239-249 (2013).

${ }^{55}$ S. N. Oskouie, Z. Yang, and B. Wang, "Study of passive plume mixing due to two line source emission in isotropic turbulence," Phys. Fluids 30, 075105 (2018).

${ }^{56}$ W. D. Baines and J. F. Keffer, "Entrainment by a multiple source turbulent jet," Adv. Geophys. 18, 289-298 (1975).

${ }^{57}$ L. Pera and B. Gebhart, "Laminar plume interactions," J. Fluid Mech. 68, 259-271 (1975).

${ }^{58}$ M. Gregoric, L. R. Davis, and D. J. Bushnell, "An experimental investigation of merging buoyant jets in a crossflow," J. Heat Transfer 104, 236-240 (1982).

${ }^{59}$ R. W. Macdonald, R. K. Strom, and P. R. Slawson, "Water flume study of the enhancement of buoyant rise in pairs of merging plumes," Atmos. Environ. 36, $4603-4615$ (2002).

${ }^{60} \mathrm{D}$. Contini and A. Robins, "Experiments on the rise and mixing in neutral crossflow of plumes from two identical sources for different wind directions," Atmos. Environ. 38, 3573-3583 (2004).

${ }^{61}$ C. Cenedese and V. M. Gatto, "Impact of two plumes' interaction on submarine melting of tidewater glaciers: A laboratory study," J. Phys. Oceanogr. 46, 361-367 (2016).

${ }^{62}$ Z. He, W. Zhang, H. Jiang, L. Zhao, and X. Han, "Dynamic interaction and mixing of two turbulent forced plumes in linearly stratified ambience," J. Hydraul. Eng. 144, 04018072 (2018).

${ }^{63}$ M. R. Mokhtarzadeh-Dehghan, C. S. König, and A. G. Robins, "Numerical study of single and two interacting turbulent plumes in atmospheric cross flow," Atmos. Environ. 40, 3909-3923 (2006).

${ }^{64}$ N. B. Kaye and P. F. Linden, "Coalescing axisymmetric turbulent plumes," J. Fluid Mech. 502, 41-63 (2004).

${ }^{65}$ C. Cenedese and P. F. Linden, "Entrainment in two coalescing axisymmetric turbulent plumes," J. Fluid Mech. 752, R2 (2014).

${ }^{66}$ G. G. Rooney, "Merging of a row of plumes or jets with an application to plume rise in a channel," J. Fluid Mech. 771, R1 (2015).

${ }^{67}$ G. G. Rooney, "Merging of two or more plumes arranged around a circle," J. Fluid Mech. 796, 712-731 (2016).

${ }^{68}$ N. B. Kaye and P. Cooper, "Source and boundary condition effects on unconfined and confined vertically distributed turbulent plumes," J. Fluid Mech. 850, 1032-1065 (2018).

${ }^{69}$ Y. Zhou, K. Nagata, Y. Sakai, and T. Watanabe, "Dual-plane turbulent jets and their non-Gaussian velocity fluctuations," Phys. Rev. Fluids 3, 124604 (2018). 
${ }^{70}$ Y. J. Suzuki, T. Koyaguchi, M. Ogawa, and I. Hachisu, "A numerical study of turbulent mixing in eruption clouds using a three-dimensional fluid dynamics model," J. Geophys. Res.: Solid Earth 110, B08201, https://doi.org/10.1029/2004jb003460 (2005).

${ }^{71}$ Y. J. Suzuki and T. Koyaguchi, "Numerical determination of the efficiency of entrainment in volcanic eruption columns," Geophys. Res. Lett. 37, L05302, https://doi.org/10.1029/2009gl042159 (2010).

${ }^{72} \mathrm{M}$. Tveitereid and D. S. Riley, "Nonparallel-flow stability of an axisymmetric buoyant plume in a coflowing uniform stream," Phys. Fluids A 4, 2151-2161 (1992).
${ }^{73}$ E. J. List, "Mechanics of turbulent buoyant jets and plumes," in Turbulent Buoyant Jets and Plumes, edited by W. Rodi (Pergamon, 1982), pp. 1-68.

${ }^{74}$ J. S. Turner, Buoyancy Effects in Fluids (Cambridge University Press, Cambridge, 1973).

${ }^{75}$ W. M. Haynes, D. R. Lide, and T. J. Bruno, CRC Handbook of Chemistry and Physics: A Ready-Reference Book of Chemical and Physical Data, 97th ed. (CRC Press, 2016).

${ }^{76}$ T. H. Shih, W. W. Liou, A. Shabbir, Z. Yang, and Z. Jiang, "A new $k-\varepsilon$ eddy viscosity model for high Reynolds number turbulent flows," Comput. Fluids 24, 227-238 (1995). 